\title{
Global Anomalies on Orbifolds
}

\author{
Daniel S. Freed ${ }^{1}$ and Cumrun Vafa ${ }^{2}$ \\ ${ }^{1}$ Department of Mathematics, Massachusetts Institute of Technology, Cambridge, MA 02139, \\ USA \\ 2 Department of Physics, Harvard University, Cambridge, MA 02138, USA
}

\begin{abstract}
We consider global anomalies for heterotic string theory formulated on orbifolds. The vanishing of certain characteristic classes in group cohomology provides sufficient conditions for the absence of global anomalies. For abelian orbifolds level matching implies these cohomology conditions, so suffices for the absence of anomalies. For nonabelian orbifolds level matching does not suffice, and there are additional constraints. We give some examples to illustrate these new constraints.
\end{abstract}

The present paper continues the discussions of global anomalies in string theory undertaken in [1] and [2, Sect. 4] to further investigate anomalies on orbifolds. ${ }^{1}$ From a mathematical point of view this study provides a beautiful, explicit example in the theory of determinant line bundles. But as the results are primarily of interest to physicists, we begin our introduction from that perspective.

String theories have been under intensive investigation in the last few years due to their potential for encompassing all known physics. The ultimate aim of the string theories will be realized only after certain issues are resolved. The most important question in this regard is how to obtain a four dimensional theory out of superstrings which are consistent only in ten dimensions.

Physicists have approached this question by assuming that the physical spacetime is actually ten dimensional, six dimensions of which are so tiny that they have thus far escaped detection (this is the Kaluza-Klein approach). This means that the space-time is assumed to be the product of four dimensional Minkowski space times a six dimensional compact manifold $M$. The main problem then in making predictions, in the context of string theory, for the so far observable four dimensional theory, is to determine $M$. There are severe restrictions on $M$ coming

The first author is partially supported by an NSF Postdoctoral Research Fellowship. The second author is supported in part by the NSF contract no. PHY 82-15249, and in part by a fellowship from the Harvard Society of Fellows

${ }^{1}$ Following the usual nomenclature in the Physics literature we refer to the quotient $M / G$ of a smooth manifold $M$ by a finite group action as an "orbifold." Here we restrict our use of this word to these quotient spaces; we do not consider the more general class of spaces which are locally quotients of this type 
from string theory, the most well-known of which is preservation of conformal invariance of the relevant non-linear sigma model (which follows from tree level equations of string theory). Not all the conditions for consistency of strings propagating in a space $M$ are known.

Certain choices of $M$, namely tori (with flat metrics), are simple and possibly fully consistent; string propagation on them is exactly solvable and the theory passes all the consistency checks known thus far. In fact tori appear in a very natural way in the bosonic formulation of the heterotic strings [3]. It seems, therefore, as a good starting guess to take $M$ to be a six dimensional torus. Unfortunately, tori do not give rise to a realistic (chiral) four dimensional theory. However, by considering strings propagating on the quotient of a torus by a finite isometry group, one obtains potentially realistic chiral string models [4] which are essentially as easily described and almost as "natural" as the toroidal compactifications. These quotients are in general not even manifolds, because the group may not act freely on the torus (i.e., it may have fixed points), and the resulting space is known as an orbifold. The singularities of orbifolds are harmless for the description of string propagation, whereas they would be problematic for the propagation of point particles.

In this context the question arises as to the consistency of strings propagating on arbitrary orbifolds, ${ }^{2}$ and in particular the absence of global anomalies, which would otherwise spoil the consistency of the theory. Absence of global anomalies in this case means that under global diffeomorphisms of the string worldsheet, which is a Riemann surface, the computations of physical processes do not get affected.

The conditions for the absence of anomalies for compactifications on smooth manifolds were first addressed in [9] where, motivated by some examples [10], it was suggested that the vanishing of (half) the difference of the integral first Pontrjagin classes of certain bundles (together with the condition that relevant bundles be spin bundles) implies the absence of global anomalies. In [2] it was proved that these conditions do indeed suffice to demonstrate the absence of global anomalies for smooth compactifications.

The question of global anomalies for orbifolds has been studied in the case where the Riemann surface is a torus (the 1-loop case) [1], where it was shown that "level matching conditions" [cf. (1.3), (1.4)] are necessary and sufficient to guarantee modular invariance at 1-loop. [In this case modular transformations are elements of $S L(2, Z)$, describing the action of global diffeomorphisms on the first homology of the torus.]

In this paper we address the question of global anomalies for orbifolds beyond one loop, i.e., for arbitrary Riemann surfaces. We will show that the level matching conditions are both necessary and sufficient for modular invariance to all loops for abelian orbifolds (tori modded out by abelian isometry groups). For non-abelian orbifolds the level-matching conditions are still necessary, but they are no longer sufficient to guarantee modular invariance - new conditions arise. Indeed, as in the smooth case the modular invariance can be stated in cohomological terms: The

\footnotetext{
${ }^{2}$ Although we only consider (symmetric) orbifolds in this paper, the same cohomology conditions apply to asymmetric orbifolds [5] in the fermionic formulation [6-8]
} 
vanishing of the second Stiefel-Whitney classes of the point group representations together with the vanishing of the difference of one-half the first Pontrjagin classes suffice to guarantee the absence of any anomalies (Corollary 7.7). For abelian orbifolds these cohomology conditions are also necessary; the level matching conditions imply the vanishing of the relevant cohomology class. In the nonabelian case, motivated by physical reasoning (Sect. 3) and a few mathematical examples (Sect. 8), we raise a question about the detectability of 4 dimensional characteristic classes of group representations by surface bundles over a circle.

On the mathematical side Corollary 5.7 is an application of the main theorem in [2, Sect. 4]. In fact, the earlier computations of the second author [1] were part of the motivation there. The explicit formulae for the Dirac determinants, and the transformation laws which result, match the topological considerations perfectly. Of course, it is the power of the topological methods that they both apply to all Riemann surfaces simultaneously (i.e., to all orders in perturbation theory) and also to any curved spacetime, where explicit formulae are usually unavailable. For example, our main result is true for any quotient space $M / G$ - the manifold $M$ need not be a torus. The cohomology of finite groups is extremely difficult to compute, and the characteristic classes of representations are even more mysterious. The question referred to above, which is born out of a mix of mathematical and physical intuitions, but owes most to the physics, is phrased in purely mathematical terms at the end of Sect. 8.

The mathematics herein may be unfamiliar to physicists. An expository account of determinant line bundles was given in [2, Sect. 1], and some topics related to integral characteristic classes were treated in [2, Sect. 3]. ${ }^{3}$ To treat orbifolds we introduce equivariant quotients. While this move is somewhat obvious to the mathematician, it may be unfamiliar to the physicist. ${ }^{4}$ In Sect. 4 we provide some motivation for our setup. Out of this discussion emerges a $G$ symmetry on the space of maps (so ultimately for our flat orbifolds only on the space of representations). The reader should compare with the considerations of Sect. 3 , where additional reasons for this symmetry are described.

The modifications to [2, Sect. 4] necessary to treat orbifolds are routine. These are explained in Sect. 5. We specialize the results to the flat orbifolds that form our main object of study. The underlying symmetry group $G$ is a discrete subgroup of the Euclidean group in 6 dimensions. This space group is supposed to contain a full lattice $\Gamma$, so that the quotient point group $P=G / \Gamma$ is finite. Our first result in Sect. 7 states that cohomology conditions on representations of $P$ suffice to conclude that global anomalies cancel. These conditions are easy to compute for cyclic groups, where they are simply the level matching conditions discussed above. For representations of abelian groups the first Pontrjagin class $p_{1}$ is determined by restricting to cyclic subgroups, which proves the sufficiency of level matching conditions (to all orders) on abelian orbifolds. Next we calculate the value of $p_{1}$ on subgroups which geometrically are images of a torus bundle over a circle. In group theoretic terms, if $A \in S L(2 ; \mathbb{Z})$ then we form the semi-direct product

\footnotetext{
${ }^{3}$ The accounts in $[12,10]$ may also be helpful. Witten describes the characteristic class " $\frac{1}{2} p_{1}$ " in $[12]$

${ }^{4}$ Note, however, that classifying spaces were already introduced in [1], where they were used to determine phase ambiguities (discrete torsion) in string amplitudes on orbifolds
} 
$K_{A}=\mathbb{Z} \times(\mathbb{Z} \times \mathbb{Z})$, where the generator acts by the transformation $A$. This group carries a canonical 3-dimensional homology class, and for homomorphisms $K_{A} \rightarrow P$ we calculate the first Pontrjagin class on the image of this homology class. Formula (7.23) duplicates (2.3), which is computed from explicit expressions for the determinants and the transformation law for the theta function. There is yet a third derivation of this expression (as the value of a Pontrjagin class) using ChernSimons invariants; it generalizes an argument of Witten [11]. We omit it. The refinements of these formulas necessary to express half the first Pontrjagin class (for representations which lift to the spin group) are also given.

Although our work is aimed at a very specific physical problem, we hope that the ideas will find wider application. It seems that the transformation laws for modular forms are topological. For example, a recent paper of Atiyah [13] relates the transformation law for the logarithm of the Dedekind eta function to Witten's global anomaly formula and to topological and group theoretic invariants. The formulas we obtain here express the transformation law for Klein forms as half a first Pontrjagin class (e.g. [14]). ${ }^{5}$ We have similarly expressed a certain transformation of theta functions [cf. (2.3), (7.44), (7.45)]. Clearly there should be a unified treatment for general transformation laws of modular forms. We remark that our computations in this paper are made under the assumption that the second StiefelWhitney class vanishes. Without that assumption the global anomaly formula leads to a computation in $K$-theory (cf. [15]) which will duplicate the more precise formulas one sees in books on modular forms. Orbifolds arise in the theory of the monster group. The moonshine module constructed in [16] can be viewed as a toroidal orbifold, where the lattice defining the torus is the Leech lattice, and each point $x$ on the torus is identified with $-x$. There is probably a connection between our characteristic class conditions and orientation in "elliptic cohomology," a new generalized cohomology theory whose main ingredient is a ring of modular forms $[17,18]$. Also, elliptic genera can be explicitly computed on orbifolds.

Expediency demands that we present our work in two stages: physics followed by mathematics. We hope that this organizational confrontation does not obscure the harmony and interaction of the two viewpoints. In Sect. 1 we review the setup for toroidal orbifolds as well as the level matching conditions. In Sect. 2 we show by direct computation that for cyclic orbifolds the level matching conditions which were found to be necessary for anomaly cancellations at one loop [1] are indeed sufficient for the absence of global anomalies on an arbitrary Riemann surface. We describe a new anomaly for nonabelian orbifolds in Sect. 3. It is detectable by a computation at one loop - the Riemann surface being a torus - and its meaning is best understood through either the operator (Hamiltonian) formulation of the theory, or by the requirement of having well-defined amplitudes at two loops and the degeneration of the 2-loop surface to two tori. Equivariant cohomology is introduced in Sect. 4; it provides the proper language to describe anomalies on orbifolds. In Sect. 5 we describe determinant bundles arising for the heterotic strings and adapt the arguments in [2] to the orbifold case. The constructions essentially go through with the relevant cohomology classes replaced by equivariant cohomology classes. In Sect. 6 we derive the cohomology conditions

${ }^{5}$ We are indebted to Glen Stevens and Joe Silverman for pointing out this reference 
from a slightly different point of view, which applies only to toroidal orbifolds. We hope it provides physicists with a more accessible derivation of the cohomology conditions. There we also prove that level matching conditions on cyclic orbifolds suffice to guarantee modular invariance at all loops. In Sect. 7 we undertake computations in group cohomology. We show that for orbifolds with abelian point groups, level matching suffices for anomaly cancellation to all loops. We also derive the explicit formulas mentioned above. We conclude with some examples in Sect. 8 .

\section{Review of Orbifolds}

In this section we review the setting for orbifolds. Generally an orbifold is a space which is locally $R^{d} / G$ where $G$ is a discrete group. More specifically, in compactifying $d$ dimensions, we consider a subgroup of the $d$ dimensional Euclidean group (in the Euclidean version of heterotic string), which includes a subgroup isomorphic to $Z^{d}$. Such a group $G$ is called a ( $d$-dimensional) space group. $Z^{d}$ defines a lattice $\Gamma$ in $R^{d}$. Therefore $T=R^{d} / \Gamma$ is a $d$ dimensional torus. The quotient $P=G / \Gamma$ is a finite subgroup of $S O(d)$ which is called the point group. The orbifold can be equivalently viewed as $T / P$. The case of phenomenological interest in physics is the compactification of the ten dimensional space to four dimensions, which corresponds to taking $d=6$.

To completely specify the heterotic string compactification, we should in addition describe the gauge bundles which are over the orbifold. We shall assume that the gauge bundle has holonomy $S O(16) \times S O(16)$ for the $E_{8} \times E_{8}$ theory, and $S O$ (32) for the $\operatorname{Spin}(32) / Z_{2}$ theory. We need this assumption in order to use the fermionic formulation of the heterotic string. To specify the bundles is tantamount to considering homomorphisms $G \rightarrow S O(32)$ [for the sake of definiteness we shall consider the $\operatorname{Spin}(32) / Z_{2}$ theory]. So a general element of the orbifold group can be written as

$$
g=(\theta, v, \phi(\theta, v)),
$$

where $\theta$ is a 6-dimensional rotation, $v$ is a translation in 6 dimensions, and $\phi(\theta, v)$ is an element of $S O(32)$.

The simplicity of the construction of the orbifolds allows one to construct the states in the string theory explicitly. Also, the modification of the path-integral of the standard theory to give the orbifold theory is very simple. To describe that we note that the physical fields in the heterotic strings includes left- and right-moving (holomorphic and anti-holomorphic) fields $x^{\mu}$ describing the space-time coordinate, tangents of the space-time coordinates, specified by right-moving fermionic fields $\psi^{\mu}$, and the 32 real (16-complex) left-moving fermionic fields $\lambda^{I}$ giving rise to $S O(32)$ gauge symmetry. When doing a path-integral we have to consider a Riemann surface with these fields defined on it (describing the immersion of the Riemann surface in the space-time, and the fermionic currents) and integrate over all the fields in the theory. In the standard heterotic string before compactification, one takes the bosonic coordinate $x^{\mu}$ to be periodic as we go around non-trivial cycles of the Riemann surface, and sum over all spin structures of the fermions (i.e., pairs of spin structures). In other words, the bosonic variables are sections of a trivial bundle, and the fermionic variables are sections of spin bundles (tensored 
with trivial bundle). However, when considering the orbifold compactification we are identifying $(x, \psi, \lambda)$ with

$$
g \cdot(x, \psi, \lambda)=(\theta x+v, \theta \psi, \phi(\theta, v) \lambda),
$$

and so we need not take the boundary conditions on the fields to be periodic. In fact, the string variables can now be a section of the $G$ bundles (tensored with spin bundles for fermions) over the Riemann surface, and we have to sum over all inequivalent $G$ bundles. More explicitly, if we choose a base point for the Riemann surface, and consider a canonical basis for the fundamental group of the Riemann surface denoted by $a_{i}, b_{i}$, then a $G$ bundle over the Riemann surface is specified by considering a homomorphism $\varrho$ from the fundamental group of the Riemann surface to the group $G$. This in turn is specified by the images of the generators of the fundamental group $g_{i}=\varrho\left(a_{i}\right)$ and $\tilde{g}_{i}=\varrho\left(b_{i}\right)$, such that

$$
\prod_{i}\left(g_{i} \tilde{g}_{i} g_{i}^{-1} \tilde{g}_{i}^{-1}\right)=1 \text {. }
$$

We then take the string variables to transform according to $g_{i}\left(\tilde{g}_{i}\right)$ as we go around $a_{i}\left(b_{i}\right)$ cycle.

The question arises as to whether there are any restrictions on $G$ and the homomorphism $\phi$. In fact there is one important constraint: The global diffeomorphisms of the surface should not change the path-integral integration. Since the 2-dimensional quantum field theory describing the heterotic string is chiral, in general phase ambiguities arise, giving rise to global gravitational anomalies $[19,11]$. In the context of orbifolds, we will have to consider global diffeomorphisms which preserve the $G$ bundle, and require that the path-integral does not pick up any phases under such diffeomorphisms.

This question was considered in [1] for the case when the Riemann surface has genus 1 , corresponding to a torus. In that case the $G$ bundle is specified by two commuting elements $(g, h)$ giving the boundary conditions for string variables. If we denote the complex structure of the torus by the complex parameter $\tau$, for a single complex fermion, with boundary conditions twisted by $g=e^{2 \pi i u}$ in the $\tau$ direction and $h=e^{2 \pi i v}$ in the other direction, the (chiral) fermionic determinant is given by

$$
d(u, v ; \tau)=e^{-i \pi(u v-u)} q^{\left(v^{2}-v+1 / 6\right) / 2} \prod_{1}^{\infty}\left(1-q^{n-v} e^{2 \pi i u}\right)\left(1-q^{n+v-1} e^{-2 \pi i u}\right),
$$

where $q=e^{2 \pi i r}$. The overall phase is ambiguous as has been discussed in [1]. This is called the Klein form [20]. The global diffeomorphism group of a torus is $S L(2, Z)$, and under an element of $S L(2, Z)$, the boundary requirement that there be no phase under this transformation is the following: For each element $g \in G$ of finite order $g^{n}=1$, we can write $\theta$ and $\phi(\theta, v)$ in a diagonal basis with eigenvalues $e^{2 \pi i r_{i} / n}$, and $e^{2 \pi i s_{k} / n}$. The condition is that if $n$ is even,

$$
\sum r_{i}=\sum s_{k}=0 \bmod 2, \quad \sum\left(r_{i}\right)^{2}=\sum\left(s_{k}\right)^{2} \bmod 2 n,
$$

and when $n$ is odd

$$
\sum\left(r_{i}\right)^{2}=\sum\left(s_{k}\right)^{2} \bmod n
$$


(These conditions have also been found to be necessary in constructing units for modular forms in [14] which is essentially why they arise here.) In the string Hilbert space construction, these conditions guarantee that left- and right-moving Hilbert spaces have infinitely many energy levels matching. We shall refer to these conditions in the following as the level matching conditions.

In the following sections we show that these conditions suffice to prove global diffeomorphism invariance (modular invariance) to all orders in perturbation theory, i.e., for any Riemann surface, in the case where the point group $P$ is abelian. In particular by explicit computation we prove in the next section that when $P$ is cyclic, these conditions suffice for modular invariance. In the case that $P$ is nonabelian, even though necessary, these conditions may not be sufficient to guarantee modular invariance to all orders. We will show that there is an additional constraint, which can be phrased in terms of computations done in one-loop, which for the non-abelian case, may turn out to suffice to guarantee modular invariance to all orders.

\section{Higher Loop Modular Invariance}

In this section we will show by using the explicit dependence of the Dirac determinants on twisting the boundary conditions of the fields on an arbitrary Riemann surface, that for cyclic orbifolds level matching conditions are sufficient to guarantee modular invariance. This generalizes the result of the 1-loop case. By cyclic orbifolds we mean orbifolds with cyclic point groups. In the following we will ignore the fact that the space group is not cyclic even if the point group is cyclic (i.e., we ignore the structure of the space group except for its point group). However, to show that level matching is sufficient to guarantee modular invariance in this case can be done for the cyclic point group by taking our starting point to be the $d$-dimensional torus (which is manifestly modular invariant) with Wilson lines turned on, rather than $R^{d}$ (see also the discussions in Sects. 6 and 7).

So far as the global anomalies are concerned we can ignore the bosonic variables, because they contribute a positive quantity to the path integral, and so no phase ambiguities arise for them. Only the fermionic fields are relevant. As discussed in the last section, the orbifold modification of the path integral is to introduce non-trivial boundary conditions for the fields. Each fermionic field on the Riemann surface corresponds to a section of a spinor bundle. For a Riemann surface of genus $g$ there are $2^{2 g}$ spinor bundles, which are specified, after a choice of canonical cycles, by a $2 g$ tuple vector $\left(\alpha_{i}, \beta_{i}\right)$ with $\alpha_{i}$ and $\beta_{i}=0$ or $1 / 2$, and $i$ runs from 1 to $g$ (for a discussion of spinors on Riemann surfaces see [21] or [22]). The difference of two spinor bundles is the changing of the holonomy by a \pm sign, as we go around the canonical cycles $\left(a_{i}, b_{i}\right)$, and $\left(\alpha_{i}, \beta_{i}\right)$ specifies precisely the difference in the boundary conditions of the spinors referred to a particular one labeled by $(0,0)$.

Since the standard heterotic string is modular invariant [9], to check modular invariance for the orbifold case, we simply have to show that the correction to the standard theory is modular invariant, and as we discussed above, the relevant correction is the ratio of the fermionic determinant when we impose twisted boundary conditions to that of untwisted fermionic determinant. We can 
describe the twistings for an abelian group, by the phases $e^{2 \pi i \theta_{i}}$ and $e^{-2 \pi i \phi_{\imath}}$ which denote how the boundary conditions on the spinor fields get modified when going around the $a_{i}$ and $b_{i}$ cycles respectively (mathematically speaking, they describe the holonomy of the flat non-trivial bundle, and the spinor fields are sections of the tensor product of this flat bundle with a spin bundle). The ratio of the twisted fermionic determinant with spin structure $(\alpha, \beta)$ to the untwisted one has been computed in [22], and is given by ${ }^{6}$

$$
\frac{\vartheta\left[\begin{array}{l}
\alpha+\theta \\
\beta+\phi
\end{array}\right](\Omega)}{\vartheta\left[\begin{array}{l}
\alpha \\
\beta
\end{array}\right](\Omega)},
$$

where in the above formula $\vartheta$ is the well known theta function (see [23] for a discussion of it) defined by

$$
\vartheta\left[\begin{array}{l}
a \\
b
\end{array}\right]=\sum_{n} e^{i \pi(n+a) \Omega(n+a)+2 \pi i(n+a) b},
$$

where $\Omega$ is the period matrix of the Riemann surface with respect to the chosen canonical homology cycles. To check modular invariance we have to see how the above ratio transforms under global diffeomorphisms acting on the Riemann surface. In particular we have to restrict to global diffeomorphisms which preserve the spin structure $(\alpha, \beta)$, as well as the flat bundle represented by $\left(\theta_{i}, \phi_{i}\right)$.

A global diffeomorphism acts on the cycles by an $\operatorname{Sp}(2 g, Z)$ transformation. We represent the element of $\operatorname{Sp}(2 g, Z)$ by

$$
T=\left(\begin{array}{ll}
D & C \\
B & A
\end{array}\right) \in \operatorname{Sp}(2 g, Z)
$$

The phase that theta functions pick under modular transformations is well known (see [24]). In fact

$$
\vartheta\left[\begin{array}{l}
\tilde{a} \\
\tilde{b}
\end{array}\right](\widetilde{\Omega})=\varepsilon(T) e^{-i \pi \phi(a, b, \Omega)} \operatorname{det}(C \Omega+D)^{1 / 2} \vartheta\left[\begin{array}{l}
a \\
b
\end{array}\right](\Omega)
$$

with

$$
\begin{gathered}
{\left[\begin{array}{l}
\tilde{a} \\
\tilde{b}
\end{array}\right]=\left(\begin{array}{rr}
D & -C \\
-B & A
\end{array}\right) \cdot\left[\begin{array}{l}
a \\
b
\end{array}\right]+\frac{1}{2}\left[\begin{array}{c}
\left(C D^{t}\right)_{d} \\
\left(A B^{t}\right)_{d}
\end{array}\right],} \\
\phi(a, b, \Omega)=\left[a D^{t} B a+b C^{t} A b\right]-\left[2 a B^{t} C b+\left(a D^{t}-b C^{t}\right)\left(A B^{t}\right)_{d}\right],
\end{gathered}
$$

where $M_{d}$ denotes a vector formed from the diagonal entries of $M$, and $\varepsilon(T)$ is a phase which only depends on $T$. Also $\widetilde{\Omega}=(A \Omega+B) /(C \Omega+D)$. In addition it is clear

\footnotetext{
${ }^{6}$ Strictly speaking the above ratio makes sense only for even spin structures where the denominator is non-zero. For odd spin structures the phase we compute is still correct, but should be viewed as the phase which arises in performing modular transformations on the interaction amplitudes (the argument of the theta function would then not be zero and the theta function does not vanish)
} 
from the definition of the theta function that for integers $m$ and $n$,

$$
\vartheta\left[\begin{array}{l}
a+m \\
b+n
\end{array}\right]=e^{2 \pi i a \cdot n} \vartheta\left[\begin{array}{l}
a \\
b
\end{array}\right] \text {. }
$$

Under a modular transformation which preserves both the spin structure and the boundary conditions, the character of the theta function shifts by integers and so putting the two phases described above we see that the ratio of the theta functions picks up the phase

$\omega=\exp \left[-i \pi\left(\theta\left(D^{t}-2\right) B(\theta+\alpha)+\alpha\left(D^{t}-2\right) B \theta+2 \theta\left(1-D^{t}\right) A \beta-\theta\left(D^{t}-1\right)\left(A B^{t}\right)_{d}\right)\right] R$,

where

$$
R=\exp \left[-i \pi\left(\phi C^{t} A(\phi+\beta)+\beta C^{t} A \phi+2(\alpha+\theta)\left(1-D^{t}\right) A \phi+\phi C^{t}\left(A B^{t}\right)_{d}\right)\right] .
$$

To put the phase in the above form we have used

$$
A-B^{t} C-1=\left(1-D^{t}\right) A,
$$

which holds, because $D^{t} A-B^{t} C=1$ for any symplectic transformation $T$. The above formula generalizes the one discussed in [1] for the genus one case. It is not difficult to see that even though $\omega$ is complicated, $\omega^{2}$ is given by the relatively simple expression,

$$
\begin{aligned}
\omega^{2} & =\exp \left[-2 i \pi\left(-\theta B \theta+\phi C \phi+\theta\left(A-D^{t}\right) \phi\right]\right. \\
& =\exp \left[-2 i \pi\left[\begin{array}{ll}
\theta & \phi
\end{array}\right]\left(\begin{array}{rr}
0 & -1 \\
1 & 0
\end{array}\right) T\left[\begin{array}{l}
\theta \\
\phi
\end{array}\right]\right] .
\end{aligned}
$$

To show this we have made use of the condition the $T$ preserve the flat bundle, i.e.,

$$
(T-1)\left[\begin{array}{r}
\theta \\
-\phi
\end{array}\right]=0 \bmod 1 \text {. }
$$

[In this derivation, it is convenient for the even spin structures to first use an $\operatorname{Sp}(2 g, Z)$ transformation to get the spin structure $\alpha=\beta=0$.] The formulas here were calculated from the formula for the ratio of Dirac determinants. The results of Sect. 5 yield a topological expression for these transformation laws. In Sect. 7 we compute the characteristic classes involved, and the result (Theorem 7.44) agrees with (2.3). It would be quite interesting to compare (2.2) and Corollary 7.45 explicitly.

Now if we start with an arbitrary twisting of a single element $g$ of the orbifold group of finite order, by an appropriate choice of the canonical basis (which could be achieved by a modular transformation), we can assume that the twisting is along the $a_{1}$ cycle alone, and the fields pick up a $g^{r}$ transformation (for some integer $r$ ) as they go around the $a_{1}$ cycle and as they go around other cycles they remain invariant. Let $h=g^{r}$, then $h$ has also a finite order, say $n$. We shall now show that modular invariance is guaranteed if the level matching conditions are satisfied in the $h$ sector. We can choose a basis where $h$ acts diagonally. Suppose one of the 
eigenvalues is given as $e^{2 \pi i \theta}$, where $\theta=r / n$. The phase $\omega$ in this case is given by (since $R=1)$

$\exp \left[-i \pi\left(\theta\left(D^{t}-2\right) B \theta+\theta\left(D^{t}-2\right) B \alpha+\alpha\left(D^{t}-2\right) B \theta+2 \theta\left(1-D^{t}\right) A \beta-\theta\left(D^{t}-1\right)\left(A B^{t}\right)_{d}\right)\right]$,

where $\theta$ is to be thought of as a $g$ dimensional vector $(\theta, 0,0, \ldots)$.

The above phase is the contribution of one fermion. We will have to take the contribution of all fermions. In addition we recall that we have two types of fermions, the right-movers, and the left-movers. In the path-integral we will get the contribution of each determinant, remembering that the determinants of leftmovers is complex conjugate of what we have written above, and therefore contribute the inverse phase under modular transformations. Also note that the two groups of fermions may have different spin structure, and we have to consider modular transformations which respect both spin structures.

We can take the level matching conditions for the case that $n$ is even (the conditions for $n$ odd can be phrased the same way as the one for $n$ even by a change of a single twist $r \rightarrow n-r$ if necessary, and so a similar argument holds for that case). We take the eigenvalues of right- and left-movers to be $e^{2 \pi i r_{i} / n}$ and $e^{2 \pi i s_{k} / n}$. The condition for level matching implies that $\sum r_{i}=\sum s_{k}=0 \bmod 2$ and $\sum r_{i}^{2}=\sum s_{k}^{2} \bmod 2 n$.

The condition that the modular transformation preserve the $h$ twisting implies that $D_{i 1}=0 \bmod n$ for $i \neq 0$ and $D_{11}-1=B_{i 1}=0 \bmod n$ for all $i$. Since $T$ is an $\operatorname{Sp}(2 g, Z)$ matrix we have $D^{t} B=B^{t} D$, which implies by the conditions on $B_{i 1}$ and $D_{i 1}$ that $B_{1 i}=0 \bmod n$.

Putting all we have said together one can show that the above phase is identical to 1 , provided the level matching conditions are satisfied. It is also clear that the level matching conditions are necessary for this general case as well as can be seen by the corresponding result for the torus and noting that the phase under modular transformations is independent of the period matrix, and one can consider modular transformations in the limit that the Riemann surface degenerates to tori. This completes the argument that in the cyclic case the level matching conditions are necessary and sufficient to guarantee modular invariance for an arbitrary Riemann surface (i.e., to all orders in perturbation).

\section{Non-Abelian Orbifold Anomalies}

In this section we discuss the anomalies that arise in the non-abelian case. We will see that some new conditions, in addition to the level matching conditions, are needed to guarantee modular invariance in this case.

In the analysis for the 1-loop case, the case of torus, one looks at the pairs of elements $(g, h)$ of the orbifold group which commute and consider changing the boundary conditions on the torus by twisting by $g$ and $h$ in the time and space directions respectively (which we call the two directions corresponding to the two cycles of a canonical basis for the first homology). This pair determines a flat nontrivial bundle over the torus. Then one computes the partition function and considers all the $S L(2, Z)$ transformations which preserve the bundle. (i.e., the 
boundary conditions),

$$
(g, h) \rightarrow\left(g^{a} h^{b}, g^{c} h^{d}\right)=(g, h) .
$$

The requirement of level matching guarantees that there is no phase under such a transformation. This condition applies to both abelian and non-abelian orbifolds. But the fact that only commuting pairs appear in the discussion, may suggest that we have not probed the non-commutativity of a non-abelian group at 1-loop. So it would seem necessary to carry out the analysis for more than 1-loop, so that the boundary conditions (that the product of commutators be identity) allow noncommutativity. However, it is rather difficult to do an explicit computation for a non-abelian group beyond one loop.

Could we get extra conditions for non-abelian orbifolds somehow by a 1-loop computation? So far we have mainly discussed the path integral formulation of orbifolds. There is, in addition, a Hamiltonian formulation, which involves the construction of operators acting on certain Hilbert spaces. To describe the Hilbert space of strings on orbifolds [4], it is convenient to consider the string states in the covering space (the torus) on which we have a sector (a subspace of the Hilbert space) for each twisting by a group element. This means that the strings in a $g$ sector consists of loops on the torus which are closed only up to a $g$ action. In addition, we have to consider group invariant states for the loops on the torus to obtain string states on the quotient. This means that we have to operate by the projection operator $\frac{1}{N} \sum_{c \in G} c$ on the string states. The Hilbert space structure of a non-abelian orbifold group differs greatly from the abelian case. In the nonabelian case to form group invariant states one takes a linear combination of states from sectors $g$ and all other sectors conjugate to it, i.e., $c g c^{-1}$. This is because if we consider a string state in the $g$ sector, operating by the group element $c$ on that string, takes the $\mathrm{g}$ sector to the $\mathrm{cgc} \mathrm{c}^{-1}$ sector.

Let us rephrase the anomaly discussed above, in the language of operators on the Hilbert space: Level matching conditions not being satisfied in the $g$ sector means that we cannot assign an unambiguous phase to the path integral with $(g, 1)$ twisting of the torus. The ambiguity of the phase of the path-integral means that the phase of the operator $g$ is ambiguous in the identity sector $[(g, 1)$ in the operator formulation can be represented by $\operatorname{Tr} g q^{H_{L}} \bar{q}^{H_{R}}$ with $H_{L}$ and $H_{R}$ corresponding to the left- and right-moving Hamiltonians in the identity sector, and $q=e^{2 \pi i \tau}$, where $\tau$ defines the modulus of torus and $g$ represents the twist operator corresponding to the group element $g$ acting on the Hilbert space].

Summing over all twisted group elements in the $\tau$ direction has the effect of projection onto the $G$-invariant subspace of the Hilbert space. Now we see that the condition that $(g, h)$ has no phase ambiguities, means only that the part of the group invariant projection consisting of elements $g$ commuting with $h$, which therefore do not change the $h$ sector, have well defined phases. We should in addition see whether there are any phase ambiguities for the group elements $c$ which do not commute with $h$ and therefore do change the $h$ sector.

The phase of (the operator) $c$ being well defined means that any computation done in one sector should be equal to the conjugate computation done in the 
conjugate sector. This implies, in particular, that

$$
\text { path integral }(g, h)=\text { path integral }\left(c g c^{-1}, c h c^{-1}\right) \text {. }
$$

We can see this in the path integral in two ways: One way is to argue from the viewpoint of the target space, that if we have a particular space-time world sheet corresponding to $(g, h)$ boundary conditions on the torus, and if we act on the whole world sheet by $c$, then the resulting world sheet has not moved in the orbifold, and so the result of the computation should not change. However, operating by $c$ in the path integral gives a torus with new boundary conditions $\left(\operatorname{cgc}^{-1}, \operatorname{chc} c^{-1}\right)$, and we obtain the above result.

The other argument which is probably more to the point and intrinsically two dimensional, relies on invariance under local diffeomorphisms of the surface and factorization (degeneration) of the two loop amplitude. Consider the two loop amplitude, specified by $(g, h ; c, 1)$ which denotes the twisting on the four canonical cycles. Note that these twistings are well defined because they satisfy (1.4):

$$
\mathrm{ghg}^{-1} h^{-1} \mathrm{c} \cdot 1 \cdot \mathrm{c}^{-1} \cdot 1=1 .
$$

However, the computation should not depend on the choice of the base point (the intersection point of the homology cycles) which has been used to define the bundle, i.e., it should be invariant under local diffeomorphisms. In particular, we can take the base point around the cycle which corresponds to the $c$ twisting back to the starting point. In this way the twistings have all changed by conjugation with $c$, i.e., we have $\left(c g c^{-1}, c h c^{-1} ; c, 1\right)$ twisting. So we must have

$$
\text { path integral }\left(c g c^{-1}, \operatorname{chc} c^{-1} ; c, 1\right)=\text { path integral }(g, h ; c, 1) \text {. }
$$

Now we can pull the two handles away and factorization forces

$$
\text { path integral }\left(c g c^{-1}, c h c^{-1}\right)=\text { path integral }(g, h) \text {. }
$$

Could there be new conditions because of the above requirement? We can simply define the contribution of $\left(c g c^{-1}, c h c^{-1}\right)$ to be equal to that of $(g, h)$. Would we run into any contradictions? In fact we may. Similar to the abelian case, if $\left(c g c^{-1}, c h c^{-1}\right)$ could be obtained from $(g, h)$ by a modular transformation, we have no choice of phase. The phase of $\left(c g c^{-1}, c h c^{-1}\right)$ would be dictated from the requirement of modular invariance. In other words we should demand that for any pair of commuting elements $(g, h)$, under all modular transformations which give a set of conjugate boundary conditions (i.e., a conjugate bundle), that is under all elements of $S L(2, Z)$ satisfying

$$
\left(g^{a} h^{b}, g^{c} h^{d}\right)=\left(c g c^{-1}, c h c^{-1}\right),
$$

the path integral should not pick a phase. The condition (3.2) is in general not easy to meet for any commuting pairs of a non-abelian group: pairs conjugate to a given commuting pair, do not usually lie on the subspace generated by the pair as is required if (3.2) is to be satisfied. However, for many non-abelian groups (3.2) does get satisfied for some pairs. In fact we will give examples of this in Sect. 8, in which not only (3.2) is satisfied for some pairs, but also that we get phases from the corresponding modular transformation, even though the theory we start with has level matching conditions satisfied for all sectors. 
Similar to the phase formula computation done in the previous section, we can compute the change of phase in this case. Since $g$ and $h$ commute we can choose a basis in which they are both diagonal. If $c g c^{-1}$ and $c h c^{-1}$ satisfy (3.2), they must also be diagonal in this basis, and since they are conjugates they must have the same eigenvalues as $g$ and $h$ respectively [it could also be that some of the eigenvalues go to their inverses (note that we are writing only one complex eigenvalue for each conjugate pair), in which case a similar formula can be derived]. This means, that $\mathrm{cgc}^{-1}$ is the same as $g$ up to a permutation of eigenvalues, and similarly for $c h c^{-1}$ and $h$. Let us denote this permutation by $P$. We denote the eigenvalues of $g$ and $h$ by $e^{2 \pi i u^{a}}$ and $e^{2 \pi i v^{a}}$ respectively (absorbing the spin structures into the twist of the fields), with $a$ running from 1 to the dimension of the representation. Set

$$
\left[\begin{array}{l}
b_{1}^{a} \\
b_{2}^{a}
\end{array}\right]=\left(\left(\begin{array}{ll}
a & b \\
c & d
\end{array}\right)-P\right)\left[\begin{array}{l}
u^{a} \\
v^{a}
\end{array}\right] .
$$

Then the phase is given (by a computation similar to the one done in [1] which can be easily generalized to all loops similar to the last section) by

$$
\prod_{a}(-1)^{b_{1}^{a}+b_{2}^{a}+b_{1}^{a} b_{2}^{a}} e^{-i \pi\left(b_{1}^{a} v^{a}-b_{2}^{a} u^{a}\right)} .
$$

In some cases this phase is not one and renders the theory inconsistent.

The question arises as to whether there are additional conditions coming from higher loops. In principle there could be. However, one would also think that the consistency of the operator formulation should suffice for the consistency of the whole theory, because we can formulate all the interactions, at least in principle, in the operator language. In other words, the consistency of the higher loops may follow from the existence, and the consistency of the operator formulation. Having a well defined Hilbert space is one of the main obstacles in formulation of the operator formulation of the theory. The above conditions, which allow a consistent $G$-invariant projection on the Hilbert space, may be sufficient to guarantee a well-defined Hilbert space for the orbifold. This physical intuition motivates our question at the end of Sect. 8, as to whether the anomalies discussed above are the only global anomalies for orbifolds. On the other hand, there may also be other physical criteria needed for the consistency of string theory (cf., the discussion at the end of Sect. 8).

\section{Equivariant Geometry}

The spacetimes we consider are quotient spaces of a smooth manifold $M$ by a finite group action. String Theory involves maps of Riemann surfaces into $M / G$. To grasp the ideas involved start with the case of a free group action. Then the quotient space is a smooth manifold, and any map $\varphi: X \rightarrow M / G$ lifts to a map of the simply connected cover $\tilde{\varphi}: \tilde{X} \rightarrow M$. This is simplest when $X$ is a circle (the simply connected cover $\tilde{X}=\mathbb{R}$ ). Physicists are familiar with this example in connection with the Hamiltonian formulation of string theory on orbifolds (Sect. 3). Now each lift $\tilde{\varphi}$ yields a homomorphism $\varrho: \pi_{1} X \rightarrow G$ which encodes the information necessary 
to reconstruct $\varphi[\mathrm{cf} .,(4.1)]$. Pictorially, we have

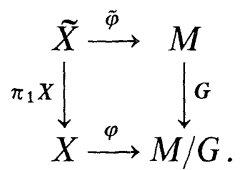

The group $G$ acts on the space of lifts with $g \in G$ sending $\langle\tilde{\varphi}, \varrho\rangle$ to $\left\langle g \circ \tilde{\varphi}, g \varrho g^{-1}\right\rangle$. In the other direction a pair $\langle\tilde{\varphi}, \varrho\rangle$ determines a map $\varphi: X \rightarrow M / G$. Note that pairs related by an element of $G$ determine the same map to $M / G$. Therefore, the space of maps from $X$ to $M / G$ is the space of pairs $\langle\tilde{\varphi}, \varrho\rangle$ up to equivalence under the $G$ action. Now if the group action is not free, then the quotient space is not a smooth manifold. The equivariant quotient construction provides for any group action a smooth space $M_{G}$ which plays the role of $M / G$ : A pair $\langle\tilde{\varphi}, \varrho\rangle$ determines a map $\varphi_{G}: X \rightarrow M_{G} \cdot{ }^{7}$ Furthermore, the geometric data used to define the fermionic (Dirac) operators extends to $M_{G}$. This justifies our use of these spaces in our mathematical formulation. In this section we quickly review the yoga associated to these equivariant matters. The reader may refer to $[25,26]$ for additional material about equivariant quotients.

There is a universal example of a $G$ action which is unique up to homotopy equivalence. It is a contractible space $E G$ on which $G$ acts freely. The quotient $B G=E G / G$ is called the classifying space of $G$.

Examples. (1) If $G=\mathbb{Z}$ then $E G$ can be taken to be the real line $R$, on which the integers act freely by translation. The quotient $B G=S^{1}$ is the circle.

(2) The circle group $G=U(1)$ acts freely on the unit sphere $S^{\infty}$ in an infinite dimensional complex Hilbert space. Furthermore, that sphere is contractible, and the quotient $B U(1)=\mathbb{C P}^{\infty}$ is the infinite complex projective space.

(3) Let $H$ be a subgroup of $G$. Then $H$ acts freely on any model for $E G$, so $B H=E G / H$. In particular, for $H=\mathbb{Z} / n \mathbb{Z} \subset U(1)$ a cyclic group we have $B(\mathbb{Z} / n \mathbb{Z})=L_{n}$ is an infinite lens space, the quotient of $S^{\infty}$ by the $\mathbb{Z} / n \mathbb{Z}$ action.

(4) It is clear that $B\left(G_{1} \times G_{2}\right)=B G_{1} \times B G_{2}$. It follows from (1) that the classifying space of a lattice is a torus. More generally, if $G=G_{1} \ltimes G_{2}$ is a semidirect product ${ }^{8}$, then $B G$ is a fiber bundle over $B G_{1}$ with fiber $B G_{2}$. For example, if $A \in S L(2 ; \mathbb{Z})$ then we can form the group $K_{A}=\mathbb{Z} \ltimes(\mathbb{Z} \times \mathbb{Z})$ with $1 \in \mathbb{Z}$ acting by $A$ on $\mathbb{Z} \times \mathbb{Z}$. The classifying space $B K_{A}$ is a 2 -torus bundle over a circle glued together by $A$ (acting as a diffeomorphism of the torus).

Notice that in each of our examples the classifying space can be approximated by a smooth finite dimensional manifold. Thus the classifying space of the circle group is approximated by a large finite dimensional complex projective space. For finite groups this also holds: Any finite group can be embedded in a unitary group (as a permutation group, via the left regular representation), and the universal space for the unitary group can be approximated by a large finite dimensional Stiefel manifold (generalizing the sphere). The quotient is a smooth manifold which approximates the classifying space of the finite group. The discrete groups we

${ }^{7} \mathrm{Up}$ to homotopy equivalence (which is harmless for our purposes)

${ }^{8}$ This means that there is an action of $G_{1}$ on $G_{2}$, and the multiplication in $G$ is defined by

$$
\left\langle g_{1}, g_{2}\right\rangle \cdot\left\langle g_{1}^{\prime}, g_{2}^{\prime}\right\rangle=\left\langle g_{1} \cdot g_{1}^{\prime}, g_{2} \cdot g_{1}\left(g_{2}^{\prime}\right)\right\rangle
$$


consider are semidirect products of a finite group and a lattice, so their classifying spaces can also be approximated by smooth manifolds. If we are willing to work directly with infinite dimensional spaces, then it is clear that the classifying spaces involved are themselves smooth manifolds.

If $M$ is a smooth manifold on which $G$ acts smoothly, then $G$ acts freely on the product $E G \times M$. The equivariant quotient is defined by $M_{G}=(E G \times M) / G$. This is a smooth manifold which fibers over the classifying space $B G=E G / G$ with fiber $M$. For $M$ a point with the trivial $G$ action we just obtain the classifying space itself. Thus in the equivariant theory the classifying space $B G$ plays the role of a point. When $G$ acts freely $M_{G}$ fibers over $M / G$ with contractible fibers $E G$. In that case the equivariant quotient is homotopy equivalent to the usual quotient. Furthermore, the geometric structures we will consider on the equivariant quotient are "constant" in the $E G$ directions, and so agree with geometric structures on the ordinary quotient. We could have achieved this with any free $G$ action, but we use the universal free $G$ action to retain all of the information encoded in the $G$ action on $M$. (The reader might contemplate what happens if we use instead the simplest free $G$ action, namely left multiplication on $G$.) For this reason the equivariant quotient is the proper generalization of the ordinary quotient.

The equivariant cohomology of the $G$-space $M$ is defined to be the ordinary cohomology of the equivariant quotient. We write $H_{G}^{*}(M)=H^{*}\left(M_{G}\right)$ to express this relationship. Note that $H_{G}^{*}(M)$ is a module over the equivariant cohomology of a point $H_{G}^{*}(p t)=H^{*}(B G)$, since $M_{G}$ fibers over $B G$. When $G$ is discrete we can identify $H^{*}(B G)$ with the group cohomology $H^{*}(G)$.

Now suppose that $E \rightarrow M$ is a vector bundle over $M$ and that we are given a lift of the $G$ action to $E$. Then $E_{G} \rightarrow M_{G}$ is a vector bundle over $M_{G}$ which plays the role of the quotient bundle. The restriction of $E_{G}$ to a fiber $M$ of $M_{G} \rightarrow B G$ is the original vector bundle $E$. The characteristic classes of $E_{G}$ in $H_{G}^{*}(M)$ are the equivariant characteristic classes of the bundle $E$. When $M$ is a point, a $G$-vector bundle amounts to a representation $\varrho$ of $G$, and its equivariant characteristic classes live in $H^{*}(B G)$. They are the characteristic classes of the representation $\varrho$.

To simplify our review of equivariant geometry, assume that $G$ is a discrete group. The general principle in this: Any $G$-invariant geometric structure on $M$ is promoted to a (partial) geometric structure on $M_{G}$. As a first example consider a metric on $M$ which is invariant under the group action. This pulls back to a partial metric on $E G \times M$ via projection onto $M$. As the pulled back partial metric is invariant under $G$, it passes to a partial metric on the quotient $M_{G}$, i.e., to a metric along the fibers of $M_{G} \rightarrow B G$. If a $G$-vector bundle $E \rightarrow M$ is endowed with a $G$ invariant metric, then the same discussion shows how to construct a metric on $E_{G} \rightarrow M_{G}$. A $G$-invariant connection on $E$ lifts to a connection on $E \rightarrow E G \times M$, which is flat in the $E G$ directions. The connection is invariant under the action of the discrete group $G$ and so passes to a connection on the quotient $E_{G} \rightarrow M_{G}$. For unitary connections we can apply the Chern-Weil homomorphism to construct closed differential forms on $M_{G}$ which represent the equivariant cohomology classes of $E$. Differential forms on $M$ which are $G$-invariant pass to differential forms on the equivariant quotient. Of course, the Chern-Weil forms on the equivariant quotient come in this way from the $G$-invariant Chern-Weil forms on $M$. 
We turn now to orbifolds. The orbifolds we consider are of the form $M / G$, where $G$ is a discrete group acting smoothly on the smooth manifold $M .{ }^{9}$ In String Theory we need to define what we mean by a map of a Riemann surface into the orbifold. More generally, we define a map $\varrho: X \rightarrow M / G$ from an arbitrary smooth manifold $X$ into the orbifold ${ }^{10}$ to be a smooth map $\tilde{\varphi}: \tilde{X} \rightarrow M$ of the simply connected cover of $X$ into $M$ together with a homomorphism $\varrho: \pi \rightarrow G$ of the fundamental group $\pi=\pi_{1}(X)$ into $G$ satisfying

$$
\tilde{\varphi}(\tilde{\sigma} \cdot h)=\varrho(h)^{-1} \tilde{\varphi}(\tilde{\sigma}), \quad \tilde{\sigma} \in \tilde{X}, \quad h \in \pi .
$$

(In String Theory, when $X$ is a Riemann surface this amounts to having string variables which are transformed by $\varrho$ as one travels around the nontrivial cycles of the surface.) The representation $\varrho$ defines a map $\psi: X \rightarrow B G$ (up to homotopy equivalence), which lifts to a $G$-equivariant map $\tilde{\psi}: \tilde{X} \rightarrow E G$. Thus $\tilde{\psi} \times \tilde{\varphi}: \tilde{X} \rightarrow E G \times M$ is $G$-equivariant and so passes to a map $\varphi_{G}: X \rightarrow M_{G}$. Conversely, given $\varphi_{G}: X \rightarrow M_{G}$, we determine a map $\varphi: X \rightarrow M / G$ by composition with the projection $M_{G} \rightarrow M / G$. We identify two maps into $M_{G}$ if there is an element of $G$ which conjugates one into the other. Namely, conjugation by an element of $G$ defines a map $B G \rightarrow B G$, since conjugation is a group homomorphism, and this lifts to $M_{G} \rightarrow M_{G}$. We define the space of maps $\operatorname{Map}(X, M / G)$ to be the quotient of $\operatorname{Map}\left(X, M_{G}\right)$ by this action. Of course, the latter space is too big, due to flabbiness in the $B G$ direction, but it will suffice for our topological applications. The reader should check that this definition works correctly when $G$ acts freely on $M$.

In the String Theory setup $M$ carries a metric and there are bundles $E \rightarrow M$ with metrics and connections; all of the data is assumed to be $G$-invariant. Associated to a map of the Riemann surface $\varphi: \Sigma \rightarrow M / G$ are certain Dirac determinants. The preceding considerations show that any of the lifted maps $\varphi_{G}: \Sigma \rightarrow M_{G}$ defines the same Dirac determinants, using the induced bundles and geometric data over the equivariant quotient $M_{G}$. This is our starting point for a mathematical treatment of global anomalies on orbifolds.

\section{Anomalies and Determinant Bundles}

The relationship between anomalies and the determinant line bundle, first describe by Atiyah and Singer [27], has been thoroughly discussed in recent literature. The space of bosonic fields $Y$ in some theory parametrizes a family of chiral Dirac operators $D$. Path integral quantization requires that the action be integrated over both the fermions and the bosons. Fermionic integration yields det $D$, which must then be integrated over the Bose fields $Y$. But det $D$ comes naturally as a section of a line bundle $\mathscr{L} \rightarrow Y$. Since $Y$ is presumed to have a measure, we must make sense of $\operatorname{det} D$ as a function on $Y$ to perform the integration. The obstruction to this is the nontriviality of $\mathscr{L}$ - the anomaly. The determinant line bundle carries a natural metric [28] and unitary connection [29]. Now the anomaly question becomes geometric: Does $\mathscr{L}$ admit a global nonzero section which is covariant constant? The obstructions are also geometric. The local anomaly is the curvature of $\nabla^{(\mathscr{L})}$

${ }^{9}$ Alternatively, they are of the form $T / P$ for a finite group $P$

${ }^{10}$ In String Theory $X=\Sigma$ is a Riemann surface 
and the global anomaly is the holonomy. Roughly speaking, one result in [30] states that the families index theorem holds on the level of differential forms: the curvature of $\mathscr{L}$ is obtained by plugging appropriate differential forms into the usual index formula. The holonomy formula was first derived by Witten [11]; it involves a certain limit of $\eta$-invariants. These issues are reviewed in [2, Sect. 1].

The geometric family of Dirac operators is specified by certain data. There is a parameter space $Y$ and a family $X_{y}$ of manifolds parametrized by $y \in Y$ which fit together into a smooth fibering $Z \stackrel{X}{\rightarrow} Y$. The fibers $X_{y}$ are compact Riemannian manifolds with a spin structure consistently defined over the family. We need to parallel transport spinor fields between fibers, and so specify horizontal complements to the vertical directions. Any external fields in the theory are represented by sections of a Hermitian vector bundle $E \rightarrow Z$ with connection. Finally, we must specify the exact combination of Dirac operators which occur.

In [2, Sect. 4] heterotic strings on smooth spacetimes were considered. (Global anomalies for these theories were first considered by Witten [9].) We recall the basic result, altering the discussion slightly. Here we use the $S O(32)$ theory (rather than the $E_{8} \times E_{8}$ theory), and real manifolds and real vector bundles (as opposed to complex manifolds and complex vector bundles). ${ }^{11}$ As compensation we take the square root of the complex Dirac determinants defined in the theory. This is justified by the fact that on a two dimensional Lorentz manifold the Dirac operator is real (there are Majorana-Weyl spinors), whereas the Riemannian Dirac operator is complex. On the other hand, the Riemannian operator is complex skew-adjoint, and this allows us to define a canonical square root of the determinant. The holonomy theorem of [30] can be refined for this square root. We use this implicitly below. ${ }^{12}$

The basic data for the heterotic string is the following.

Data 5.1. (1) $\Sigma$, a closed Riemann surface.

(2) $\operatorname{Met}(\Sigma)$, the space of metrics on $\Sigma$.

(3) $\operatorname{Spin} \operatorname{Str}(\Sigma)$, the set of spin structures on $\Sigma$. It is a finite set of $2^{2 g}$ elements for a Riemann surface of genus $g$.

(4) The spacetime $M$, a smooth oriented manifold of dimension 10 with a fixed Riemannian metric.

(5) $V$, a rank 32 oriented real vector bundle over $M$. It is equipped with a metric and compatible connection.

(6) $H \in \Omega^{3}(M)$, a 3 -form which satisfies

$$
d H=\frac{1}{2} p_{1}\left(\Omega^{(M)}\right)-\frac{1}{2} p_{1}\left(\Omega^{(V)}\right) .
$$

Here $\frac{1}{2} p_{1}(\Omega)$ denotes the Chern-Weil 4-form $\frac{1}{16 \pi^{2}} \operatorname{Tr} \Omega^{2}$ associated to the skew-

${ }^{11}$ Although complex spaces were used in [2, Sect. 4], a proper treatment of the $E_{8} \times E_{8}$ theory uses real spaces and square roots as we do here. This requires some modifications (for example to the bordism calculations) which we leave for the reader's enjoyment

${ }^{12}$ The idea for this square root arose from a conversation with Ed Miller. A complete account of the square root will appear in [15]. The basic idea is that if $V$ is a $2 r$ dimensional complex vector space and $T: V \rightarrow V^{*}$ a nonsingular skew-symmetric map, then $T$ can be identified with an element $\omega_{T} \in \Lambda^{2} V^{*}$. The complex Pfaffian $\operatorname{Pfaff}(T)=\omega_{T}^{r} \in \operatorname{det} V^{*}$ satisfies $\operatorname{Pfaff}(T)^{\otimes 2}=\operatorname{det}(T)$. The two dimensional Dirac operator is complex skew-adjoint 
symmetric curvature matrix $\Omega$. [In the physics literature (5.2) appears as $d H=\operatorname{Tr} R^{2}-\operatorname{Tr} F^{2}$ up to constants. $]$

(7) $\operatorname{Map}(\Sigma, M)$, the space of smooth maps from $\Sigma$ to $M$.

From this data we form the family of Riemann surfaces

$$
\begin{aligned}
& \quad Z=\operatorname{Met}(\Sigma) \times \operatorname{Map}(\Sigma, M) \times(\operatorname{Spin} \operatorname{Str}(\Sigma))^{2} \times \Sigma / \operatorname{Diff}_{0}(\Sigma) \\
& \pi \downarrow \Sigma \\
& \quad Y=\operatorname{Met}(\Sigma) \times \operatorname{Map}(\Sigma, M) \times(\operatorname{Spin} \operatorname{Str}(\Sigma))^{2} / \operatorname{Diff}_{0}(\Sigma) .
\end{aligned}
$$

Here we have divided out by the obvious action of $\operatorname{Diff}_{0}(\Sigma)$, the connected component of the diffeomorphism group; it acts trivially on the spin structures. If we fix spin structures $\alpha, \beta$ and restrict to the corresponding components of $Y$, then we can divide out by the group $\operatorname{Diff}_{\alpha, \beta}(\Sigma)$ of diffeomorphisms preserving the triple of spin structures. The combination of Dirac-type (fermionic) operators which arises in the heterotic string is ${ }^{13}$

$$
\left(\operatorname{det} D_{\alpha, T M}\right)^{1 / 2}\left(\operatorname{det} \overline{D_{\beta, V}}\right)^{1 / 2}\left(\operatorname{det} D_{\alpha, L}\right)^{-1} \text {. }
$$

In this expression $L \rightarrow Z$ is the complex line bundle consisting of (vertical) tangent vectors along the Riemann surfaces, and $D_{\alpha, L}$ is the chiral Dirac operator (relative to the spin structure $\alpha$ ) coupled to $L$. This is the (complex) Rarita-Schwinger operator. The family of operators described in (5.4) gives rise to a determinant line bundle $\mathscr{L}$ and connection $\nabla^{(\mathscr{L})}$.

The physical connection requires a slight modification (due to the "torsion field" ${ }^{14} H_{\mu \nu \varrho}$ ). Let $e: Z \rightarrow M$ denote the natural evaluation map. Define a 1 -form $\omega$ on $Y$ by

$$
\omega=(2 \pi i) \pi_{*} e^{*} H .
$$

In other words, $\omega$ is the integral of $H$ over the image of the Riemann surface in spacetime. The physical connection is $\nabla^{(\mathscr{L})}-\omega$.

The conditions for the cancellation of all local and global anomalies involve the four dimensional integral characteristic class $\lambda$ associated to the Spin group which satisfies $2 \lambda=p_{1}$. The second Stiefel-Whitney class $w_{2}$ is defined for real vector bundles $E$. If $E$ is oriented and $w_{2}(E)=0$, then $E$ admits a spin structure. The characteristic class $\lambda(E)$ is independent of the spin structure.

Theorem 5.5 [2, Theorem 4.15]. The following are sufficient conditions for the cancellation of global anomalies:

$$
\begin{gathered}
w_{2}(M)=w_{2}(V)=0 ; \\
\lambda(M)=\lambda(V) .
\end{gathered}
$$

However, we may have to adjust $H$ (by a closed 3-form) to cancel all global anomalies.

Notice that over the reals (ii) is the cohomological content of (5.2). Condition (i) is equivalent to the assertion that $M$ and $V$ admit spinors.

\footnotetext{
${ }^{13}$ In the notation of Sect. $1, D_{\alpha, T M}$ operates on the $\psi$ fields and $\overline{D_{\beta, V}}$ operates on the $\lambda$ fields

${ }^{14}$ One can view $H$ as the torsion of a non-Riemannian connection on $T M$
} 
We can make a more precise statement. A loop in the configuration space determines a 3-manifold $K$ which fibers over the circle. It is constructed by gluing the ends of $\Sigma \times[0,1]$ together using a diffeomorphism of $\Sigma$, and it comes equipped with an evaluation map $e: K \rightarrow M$. Then there is a cohomology class $\mu \in H^{3}(M ; \mathbb{R} / \mathbb{Z})$ determined by $\lambda(M), \lambda(V)$, and the $H$ field such that the pairing $\left\langle[K], e^{*} \mu\right\rangle$ computes the holonomy of the determinant bundle around the loop. In particular, the holonomy depends only on the class of $[K]$ in $H_{3}(M)$.

The preceding setup is easily modified to accommodate orbifolds. Let $G$ be a discrete group acting on a smooth manifold $M$. We do not assume that $G$ is finite, although in our examples the singularities of $M / G$ are of finite type. The basic data (5.1) must be modified to account for the group action. ${ }^{15}$ Thus in (4) we assume that the action of $G$ on $M$ preserves the Riemannian metric. Further, we must be given a lift of the $G$ action to the bundle $V$ in (5). The latter should also preserve metrics and connections. Finally, the $G$ action should preserve the 3 -form $H$ in (6). With these assumptions we can construct the equivariant quotient $M_{G}$ together with a partial metric, in other words a metric on the equivariant tangent bundle $(T M)_{G} \rightarrow M_{G}$. Also, the equivariant bundle $V_{G} \rightarrow M_{G}$ comes equipped with metric and connection. Finally, the 3-form $H$ passes to a 3-form on $M_{G}$ satisfying (5.2) for the equivariant Pontrjagin forms.

The most significant modification comes in (7). As discussed in Sect. 4 we use $\operatorname{Map}\left(\Sigma, M_{G}\right)$ as the space of maps into the orbifold. Now the group $G$ acts as an additional discrete symmetry conjugating these maps. Thus the union of the components of configuration space for String Theory on $M / G$ corresponding to spin structures $\alpha, \beta$ is

$$
\operatorname{Met}(\Sigma) \times \operatorname{Map}\left(\Sigma, M_{G}\right) / \operatorname{Diff}_{\alpha, \beta}(\Sigma) \times G .
$$

The Riemann surface $\Sigma$ fibers over this configuration space, and the total space of that fibration maps to $M_{G}$ by evaluation. The combination of determinants (5.4) is a section of a line bundle over (5.6) (after appropriate modifications for zero modes), the line bundle is endowed with a connection, and as before the anomaly is the holonomy of the connection. Theorem 5.5 applies directly to this situation to yield

Corollary 5.7. The following conditions in the equivariant cohomology of $M$ are sufficient to guarantee the cancellation of global anomalies on the orbifold $M / G$ :

$$
\begin{gathered}
w_{2}^{G}(M)=w_{2}^{G}(V)=0 ; \\
\lambda^{G}(M)=\lambda^{G}(V) .
\end{gathered}
$$

Here $w_{2}^{G}$ and $\lambda^{G}$ are equivariant characteristic classes. However, we may have to adjust $H$ (by a $G$-invariant closed 3-form) to cancel all global anomalies.

For the flat orbifolds introduced in Sect. 2 the conditions in Corollary 5.7 reduce to conditions in group cohomology. Recall that for these orbifolds $M=\mathbb{R}^{10}$

\footnotetext{
${ }^{15}$ It may be helpful for the reader to follow these constructions in the case where $G$ acts freely on $M$. Then the equivariant constructions are superfluous and can be replaced by direct consideration of $M / G$
} 
and $G$ is a subgroup of the 6 dimensional Euclidean group Euc(6). Let

$$
\varrho: G \rightarrow \operatorname{Euc}(6)
$$

denote the inclusion; it is an affine representation of $G$. The group $G$ contains a full lattice subgroup $\Gamma$ (isomorphic to $\mathbb{Z}^{6}$ ), so that the quotient $G / \Gamma$ is a finite group $P$ contained in $S O(6)$. Let $T$ denote the torus $\mathbb{R}^{6} / \Gamma$. Thus there is an exact sequence

$$
1 \rightarrow \Gamma \rightarrow G \stackrel{j}{\rightarrow} P \rightarrow 1 \text {. }
$$

The orbifold $M / G$ is

$$
\mathbb{R}^{10} / G \simeq \mathbb{R}^{4} \times T / P
$$

Now since $\mathbb{R}^{10}$ is contractible, the equivariant quotient $M_{G}$ is homotopic to the classifying space $B G$. Furthermore, by (5.9) there is a fibration $B G \rightarrow B P$ with fiber $B \Gamma$ a 6 dimensional torus. The representation $\varrho$ defines a 6 dimensional Euclidean bundle over $M_{G} \sim B G$ which, after summing with a trivial rank 4 bundle, is the equivariant tangent bundle. Its (stable) characteristic classes, the equivariant characteristic classes of $M$, are the characteristic classes of the representation $\varrho$ in $H^{*}(B G)=H^{*}(G)$. The vector bundle $V$ is determined by a representation

$$
\phi: G \rightarrow S O(32) ;
$$

the equivariant characteristic classes of $V$ are the characteristic classes of $\phi$. Both $M$ and $V$ are flat bundles, so we can take the 3-form $H$ in (6) to be zero.

Corollary 5.12. For these flat orbifolds the global anomalies cancel if the following conditions on the characteristic classes of the representations $\varrho$ and $\phi$ hold:

$$
\begin{gathered}
w_{2}(\varrho)=w_{2}(\phi)=0 ; \\
\lambda(\varrho)=\lambda(\phi) .
\end{gathered}
$$

We will analyze these conditions in Sect. 7. Here we simply indicate the relationship of the more abstract setup of this section with the physical discussions of Sects. 1-3. We must describe loops in the configuration space (5.6). Since $M_{G}=B G$, a map from $\Sigma$ to $M_{G}$ is determined topologically by a representation $\sigma: \pi_{1} \Sigma \rightarrow G$. The space $\operatorname{Hom}\left(\pi_{1} \Sigma, G\right)$ of such representations is discrete, so a path in this space is constant. Thus a loop in the configuration space consists of a fixed representation $\sigma$; two conformal structures related by a diffeomorphism $\varphi$ of $\Sigma$ preserving the pair of spin structures $\alpha, \beta$; and an element $c \in G$ such that if $\varphi_{*}$ is the induced action of the diffeomorphism on $\pi_{1} \Sigma$, then

$$
\sigma \circ \varphi_{*}=c \sigma c^{-1} \text {. }
$$

This data yields a 3-manifold $K$ fibered over the circle (with typical fiber $\Sigma$ ) and a flat $G$ bundle over $K$.

For $\Sigma$ a torus the fundamental group $\pi_{1} \Sigma \cong \mathbb{Z} \times \mathbb{Z}$. Fix an identification. Then a representation $\sigma$ is simply a choice of two elements $g, h \in G$ which commute. Furthermore, $\varphi_{*}$ acts on $\pi_{1} \Sigma=H_{1}(\Sigma)$ preserving the intersection form, so that $\varphi_{*}$ 
is an element of $S L(2 ; \mathbb{Z})$. Relative to our fixed basis of the homology it is represented by a matrix $\left(\begin{array}{ll}a & c \\ b & d\end{array}\right)$. Equation (5.13) is equivalent to

$$
g^{a} h^{b}=c g c^{-1}, \quad g^{c} h^{d}=c h c^{-1} .
$$

This agrees with (3.2). When $c=1$ we recover (3.1). Note that even for abelian point groups the space group can be nonabelian, so we may have to consider (5.14) with nonzero values of $c$.

For a Riemann surface $\Sigma$ of genus g, let $\Gamma$ denote the mapping class group $\pi_{0} \operatorname{Diff}(\Sigma)$. Then there is an exact sequence

$$
1 \rightarrow \operatorname{Torelli}(\Sigma) \rightarrow \Gamma \rightarrow \operatorname{Sp}\left(H_{1} \Sigma\right) \rightarrow 1 .
$$

Here $\operatorname{Sp}\left(H_{1} \Sigma\right)$ is the group of automorphisms of $H_{1} \Sigma$ which preserve the intersection form. Under an identification $H_{1} \Sigma \cong \mathbb{Z}^{2 g}$ via a canonical basis, this group is identified with $\operatorname{Sp}(2 g ; \mathbb{Z})$. The elements of $\Gamma$ which act trivially on homology comprise the Torelli group. Suppose $G$ is an abelian group. Then a representation $\sigma: \pi_{1} \Sigma \rightarrow G$ is a choice of $2 g$ elements $g_{i}, h_{i}$ in $G$. A diffeomorphism $\varphi$ preserves $\sigma$ if the action $\varphi_{*}$ on homology, which is represented by $\left(\begin{array}{ll}A & C \\ B & D\end{array}\right) \in \mathrm{Sp}(2 \mathrm{~g} ; \mathbb{Z})$, satisfies a condition we write symbolically as

$$
g^{A} h^{B}=g, \quad g^{C} h^{D}=h .
$$

\section{Another View of Orbifold Anomalies}

In this section we discuss the sufficiency conditions for the absence of global anomalies on orbifolds. Whereas in the last section we were concerned with anomalies on arbitrary orbifolds, in the present section we will focus on toroidal orbifolds which have been studied in connection with finding simple models for compactification. Our approach is somewhat different from the previous section, but at the end we obtain the same necessary conditions for absence of anomalies. ${ }^{16}$ In particular, we show that the vanishing of $w_{2}$ (the second Stiefel-Whitney class) of the representation, and the vanishing of $p_{1}$, implies the absence of global anomalies, up to a potential $Z_{2}$ anomaly which is not captured by the Pontrjagin class. We also describe a characteristic class for spin bundles, which captures the full anomaly, including the potential $Z_{2}$.

In this section we will first show how, so far as anomalies are concerned, the question reduces to the point group and its (properly defined) representation. The reader should compare this with a more mathematical argument given in Sect. 7 (Corollary 7.7). Then we will use properties of classifying spaces to compute anomalies and derive sufficiency conditions for its absence, In addition we will show that for cyclic orbifolds level matching suffices to prove absence of global anomalies, by a topological argument similar to the one discussed in [9] (we generalize the argument in [9] from $Z_{2}$ to $Z_{n}$ ). The more general assertion for

${ }^{16}$ Our main aim in this section is to provide physicists with a more accessible derivation of the cohomology conditions which were derived in Sect. 5, at least for the toroidal orbifolds 
abelian orbifolds is proved in Sect. 7 using group cohomology (cf. Corollary 7.18). Some of the arguments below already appear in [9]. However, we discuss them here in the context of orbifolds and generalize some of them.

In Sect. 1 we have noted that an orbifold is defined by a space group whose elements can be written in the form

$$
g=(\theta, v, \phi(\theta, v)) .
$$

The point group $P$ consists of rotations $\theta$, such that $(\theta, *, *)$ is in the space group. Consider the elements of the form

$$
(1, v, \phi(v)),
$$

which define the lattice $\Gamma$. $\Gamma$ defines the underlying torus by the vectors $v$ and $\phi(v)$ define the Wilson lines turned on the torus (Wilson lines are flat connections). Consider the gauge fields $A$ which are in the Cartan subalgebra of the group which generate this Wilson line. This means that $\phi(v)=\exp \left(-i \int_{0}^{v} A\right)$. Now it is well known that there are no anomalies for the standard heterotic strings [9], as well as its toroidal compactifications with Wilson lines turned on [31,32]. So we can consider what changes we make in the path integral compared to this and check whether the correction factor has any anomalies. To this end we note that so far as the global anomalies are concerned only the fermions are relevant, the NSR fermions and the gauge fermions. The NSR fermions transform as $\theta$, and the gauge fermions as $\phi(\theta, v)$, for each element $g$. In comparison to the toroidal compactification we see that $g$ has the added effect of $\theta$ on NSR fermions, but $\tilde{\phi}(\theta, v)$ $=\exp \left(i \int_{0}^{v} A\right) \phi(\theta, v)$ on gauge fermions. It is easy to show that if $(\theta, v, \phi(\theta, v))$ and $(\theta, w, \phi(\theta, w))$ are both in the group, then

$$
\tilde{\phi}(\theta, v)=\exp \left(i \int_{0}^{v} A\right) \phi(\theta, v)=\exp \left(i \int_{0}^{w} A\right) \phi(\theta, w)=\tilde{\phi}(\theta, w),
$$

which implies that $\tilde{\phi}$ is independent of the shift vector and can be written as $\tilde{\phi}(\theta)$. In this way we reduce the anomaly question to the point group $P$ given by the representation

$$
(\theta, \tilde{\phi}(\theta)) \text {. }
$$

In the computations for the anomaly the phase coming from the left-mover gauge fermions appear with the opposite sign from the right-mover NSR fermions. Apart from that they are identical, and so we shall treat only one of them (or one can think of both at the same time remembering that we have an indefinite (Lorentzian) signature in the expressions below for the anomalies).

We first review the definition of the first Pontrjagin class $p_{1}$ of a group representation. As discussed in the last section, for any finite group $G$ and a real representation of it there corresponds a vector bundle over the classifying space $B G$, and the characteristic classes of the bundle are cohomology classes in $H^{*}(B G)$ $=H^{*}(G)$. In particular for each representation we have $w_{2} \in H^{2}\left(G, Z_{2}\right)$ and $p_{1} \in H^{4}(G, Z)$. Since the cohomology classes are pure torsion [i.e., the order of 
$H^{*}(G)$ is finite], by universal coefficient theorem $H^{4}(G, Z)$ is isomorphic to homomorphisms

$$
H_{3}(G, Z) \rightarrow U(1) \text {. }
$$

This means that for any element (for example $p_{1}$ ) in $H^{4}(G, Z)$, and any closed three dimensional cycle $K$ representing an element in $H_{3}(G, Z)$ we can compute a phase $e^{2 \pi i \theta}$, or equivalently a real number $\theta$ well defined up to addition of integers. We denote this by writing

$$
p_{1}[K]=\theta \bmod Z .
$$

For applications to string theory it is convenient to give an expression for $\theta$ in terms of differential forms. To do this we consider a representation of $G$ by special orthogonal groups. This means we have a homomorphism

$$
G \rightarrow S O(d) .
$$

Now suppose this representation admits a "lift" to $\operatorname{Spin}(d)$. This means that there is a homomorphism $G \rightarrow \operatorname{Spin}(d)$, which combined with the standard projection $\operatorname{Spin}(d) \rightarrow S O(d)$ gives the above homomorphism. This lift exists if and only if $w_{2}=0$. We view $G$ as a subgroup of $\operatorname{Spin}(d)$ via this homomorphism. This implies that there is a map $B G \rightarrow B$ Spin, where $B$ Spin denotes the classifying space for the spin groups. This means that we can think of $K$ as sitting in $B$ Spin via this map. Since $H_{3}(B$ Spin, $Z)=0$, this implies that $K$ can be viewed as the boundary of a four dimensional space ${ }^{17} B$ in $B$ Spin. We recall that any classifying space comes equipped with a universal bundle, and a universal connection. We will use the universal spin connection on $B$ Spin to define $\theta$. Let $F$ denote the curvature of this connection. Then ${ }^{18}$

$$
\theta=p_{1}[K]=\frac{1}{8 \pi^{2}} \int_{B} F \wedge F
$$

This expression can be equivalently viewed as the definition of $p_{1}$ (see [2, Sect. 3] to see that it is equivalent to the more standard definition of Pontrjagin class). Note that $\theta$ is well-defined up to an addition by even integers, because the difference in $\theta$ due to two different choices of $B$ amounts to the integration of the instanton density over a closed four dimensional manifold, which is even for real representations. However, $p_{1}$ is defined only modulo integers. So we see that $\theta$ defined by this integral is finer than $p_{1}$ in that it contains an additional $Z_{2}$ information. This means that there is a finer characteristic class of the $B$ Spin called $\lambda$, given by $\frac{1}{2} \theta$, which is well defined up to an addition of integers (for a more standard description of this characteristic class accessible to physicists see [12]).

Now we will describe global anomalies on orbifolds. The absence of anomalies means that under global diffeomorphisms respecting the bundle (up to conjugation) and the spin structures on the surface, the path-integral should not pick a phase. To compute global anomalies we consider a one parameter family of

\footnotetext{
${ }^{17}$ Here we use the stronger fact that $\Omega_{3}^{\text {spin }}(B$ Spin $)=0$, and therefore we choose $B$ to be a spin manifold

${ }^{18}$ Here we must assume that the map $K \rightarrow B$ Spin pulls the universal connection back to the flat $G$ connection on $K$
} 
Riemann surfaces, parameterized by $t \in[0,1]$, and identify the Riemann surfaces over $t=0$ and $t=1$ by the global diffeomorphism. In this way we obtain a three dimensional space which we call $K$. Since the global diffeomorphism respects the bundle and the spin structures, we get in addition a $G$ bundle over $K$, and $K$ admits the two spin structures inherited from the Riemann surface. The phase that the measure picks is $e^{-i \pi \eta}$, where $\eta$ is the relevant (generalized) eta function of the relevant operators in string theory. To compute $\eta$ we use the property of classifying spaces, in that any space with a bundle and connection can be viewed as sitting in the classifying space, with the connection induced from the universal connection of the classifying space (mathematically this means that every bundle and connection can be obtained by pulling back the universal bundle and connection over the classifying space, by a map to the classifying space). In this way we can view $K$ as sitting in $B G$ or, more conveniently, in $B$ Spin. Here we are assuming that $w_{2}=0$ which is equivalent to the $\bmod 2$ condition on the group elements of even order. This can also be understood because the actual gauge group is $\operatorname{Spin}(32)$, and the space time also admits spinors. Non-trivial cobordism arguments given in [12] applied to this case show that $K$ can be viewed as the boundary of a four dimensional space $B$ with spin structures and bundles extended over $B$.

We are finally in a position to compute $\eta$. By the Atiyah-Patodi-Singer index theorem [33], for any operator $D$ on $K$,

$$
\eta(D)=\operatorname{index}(D)-\int_{B} \operatorname{Ind}(D)
$$

where index $(D)$ is an integer denoting the index of the operator $D$ on $B$ and $\operatorname{Ind}(D)$ is the index density of the operator $D$ which is a polynomial in curvature of the relevant bundles (note that the operator $D$ should be extendible over $B$ to use the above formula). ${ }^{19}$ Since the toroidally heterotic strings are free from anomalies, we should see what changes in the computation of $\eta$. In fact all that changes is that we get a different contribution from the chiral Dirac fermions corresponding to the representation of the point group discussed above. We have

$$
\begin{gathered}
\eta=\Delta \eta=-\frac{1}{8 \pi^{2}} \int_{B} F \wedge F=\theta \bmod 2 Z, \\
\theta=p_{1}(K) \bmod Z .
\end{gathered}
$$

The first expression determines $\eta$ up to addition of an even integer, so we see that the total phase $e^{-i \pi \eta}$ is

$$
e^{-i \pi \eta}=e^{-i \pi \theta}= \pm e^{-i \pi p_{1}[K]}
$$

The \pm sign in the second equality is because $p_{1}[K]$ is defined only up to addition of integers. This shows that if $p_{1}=0$ the anomaly is reduced to at most a $Z_{2}$ anomaly. But if the other invariant called $\lambda$ vanishes, the anomaly is totally absent (putting in

\footnotetext{
${ }^{19}$ We can derive the above formula for the phase of the Dirac determinant by the computation done in [34] by enlarging our space, as we have done here, to consider $\operatorname{Spin}(d)$ connections, so that the global anomaly reduces to a local anomaly computation as has been done for the global $S U(2)$ anomaly [35]. Note that in this way we can bypass the identification of the phase with eta and the APS index theorem and directly obtain the phase
} 
the right-movers implies that the difference of $\lambda$ between the two representations should vanish, and that $w_{2}$ of each of the representations is trivial).

We will now show that when $G$ is cyclic level matching conditions suffice to guarantee absence of global anomalies. A different proof of this by computations in group cohomology appears in Sect. 7. We take $G=Z_{n}$. Over $K$ we have a $Z_{n}$ bundle. Consider $G \subset U(1)=S O(2)$ by mapping the generator of $G$ to $e^{2 i \pi / n}$. The $Z_{n}$ bundle over $K$ admits a flat $U(1)$ gauge field. This means that the connection has appropriate holonomies about the cycles of $K$. Suppose the representation of (leftmoving) fermions is given by $e^{2 \pi i r_{i} / n}$, with $i=1, \ldots, k$ and its complex conjugate (the dimension of the real representation is $2 k$ ). The twisted Dirac determinants is the same as that of $k$ untwisted (complex) Dirac fermions which are coupled to the $U(1)$ field with the $k$ fermionic $U(1)$ charges given by $r_{i}+a_{i} n$ for arbitrary $a_{i}$ (we can add any multiple of $n$ to the charge without changing the holonomy). We will use below our freedom in choosing any $a_{i}$. As we mentioned above, the only difference in computation of the global anomaly for the measure in the orbifold case is that of fermionic determinants. So if the $\eta$ computed on $K$ for this representation of fermions with the flat $U(1)$ gauge field is the same as that with a different representation, which does not have any twists on $K$, then there will be no anomalies. A representation of $U(1)$ which has no twists on $K$ corresponds to having charges which are multiples of $n$. The difference in the computation of the $e^{-i \pi \eta}$ for two different representations is simply given by computing the ratio of $e^{-i \pi \theta}$ for the two representations (if the $w_{2}$ vanishes, which is identical to the mod 2 conditions). This phase is therefore zero if $F \wedge F$ is identical for the two representations. This can be arranged if

$$
\sum\left(r_{i}+a_{i} n\right)^{2}=0 \bmod n^{2}
$$

(if necessary we can add equal number of representations to both sides which have charges a multiple of $n$ ). We immediately see that the condition above cannot be satisfied unless $\sum r_{i}^{2}=0 \bmod n($ and $\bmod 2 n$ for even $n)$. These are precisely the level matching conditions. If these conditions are satisfied it is indeed possible to find $a_{i}$ to satisfy the above equation, and so the anomalies cancel because of the above argument. This shows that level matching suffices to prove absence of global anomalies to all orders for a cyclic group.

Now let us consider an arbitrary finite abelian group. Any finite abelian group can be written as the product of finite number of cyclic groups $Z_{n_{1}} \times \ldots \times Z_{n_{l}}$ with $n_{i}$ dividing $n_{i+1}$ [36]. Let us consider the case when $l=2$. In that case we can repeat the above procedure, except that we have to introduce two $U(1)$ fields. $F \wedge F$ in this case contains three types of terms $F_{1} \wedge F_{1}, F_{1} \wedge F_{2}$, and $F_{2} \wedge F_{2}$, where $F_{1}$ and $F_{2}$ are the curvatures of the two $U(1)$ fields. Let the respective eigenvalues be $r_{i}^{1}, r_{i}^{2}$. The conditions that have to be solved to prove no anomalies are now given by

$$
\begin{gathered}
\sum\left(r_{i}^{1}+a_{i}^{1} n_{1}\right)^{2}=0 \bmod n_{1}^{2}, \\
\sum\left(r_{i}^{1}+a_{i}^{1} n_{1}\right)\left(r_{i}^{2}+a_{i}^{2} n_{2}\right)=0 \bmod n_{1} n_{2}, \\
\sum\left(r_{i}^{2}+a_{i}^{2}\right)^{2}=0 \bmod n_{2}^{2} .
\end{gathered}
$$


The first and third conditions can be satisfied only if there is level matching for the generators of $Z_{n_{1}}$ and $Z_{n_{3}}$ respectively. The second condition can be satisfied only if there is level matching for the product of the generators of the two groups.

Even if these conditions are satisfied, it may still not be possible to solve the above equations. We could redefine the generators to try to find solutions to the above equations if one set of generators do not give solutions to the above equations. However, we will not pursue this approach further. In Sect. 7 we will compute the relevant characteristic classes in group cohomology which implies that these conditions are sufficient even for the product of cyclic groups.

We emphasize that the arguments we used here to prove absence of global anomalies for the cyclic case are very particular to the abelian nature of the orbifold point group. In the non-abelian case we have the abstract formulation of the absence of anomalies captured by the characteristic classes $\lambda$ and $w_{2}$.

\section{Computations in Group Cohomology}

The conditions in Corollary 5.12 are stated in terms of the infinite discrete space group $G$. We begin our analysis by replacing the representations $\varrho, \phi$ with associated representations $\varrho_{P}, \phi_{P}$ of the finite point group $P$. Then we evaluate the characteristic classes $w_{2}$ and $\lambda$ on abelian groups. For representations of abelian groups we prove that these classes are completely detected by cyclic subgroups. In physical terms this says that the level matching conditions for each sector guarantee anomaly cancellation for abelian point groups. (Later in this section we prove that level matching is necessary for anomaly cancellation with any point group.) Then we calculate these characteristic classes on groups $K_{A}$ formed by adjoining an automorphism $A \in S L(2 ; \mathbb{Z})$ to $\mathbb{Z} \times \mathbb{Z}$. Topologically, such a group is a torus bundle over a circle. The formulae we obtain are related to the transformation law for the theta function (2.2).

Consider first the inclusion representation $\varrho: G \rightarrow \operatorname{Euc}(6)$. Recall that for the Euclidean group there is a split exact sequence

$$
1 \rightarrow \mathbb{R}^{6} \rightarrow \operatorname{Euc}(6) \stackrel{k}{\rightleftarrows} S O(6) \rightarrow 1 .
$$

Since $\mathbb{R}^{6}$ is contractible, the induced map $B k: B \operatorname{Euc}(6) \rightarrow B S O(6)$ is a homotopy equivalence. Now there is a commutative diagram

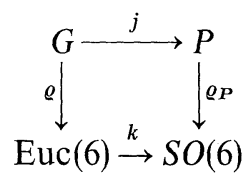

and an induced diagram on classifying spaces. The commutativity of (7.2) yields

Proposition 7.3. For any characteristic class $v$ of $S O(6)$, we have $v(\varrho)=j^{*} v\left(\varrho_{P}\right)$. In particular, this applies to $w_{2}$ and $\lambda$.

The representation $\phi: G \rightarrow S O(32)$ requires a slightly more involved argument. ${ }^{20}$ Observe that since $\Gamma$ is abelian, we can assume that $\phi(\Gamma)$ lies in the

${ }^{20}$ This argument simplifies if (5.9) is a split extension. 
usual maximal torus of $S O(32)$. Fix an identification $\Gamma \cong \mathbb{Z}^{6}$, and write an element of $\Gamma$ as $a^{1} e_{1}+\ldots+a^{6} e_{6}\left(a^{i} \in \mathbb{Z}\right)$ relative to this choice of basis. Then there exist numbers $\theta_{i}^{j}$ such that $\phi$ maps $\Gamma$ to block diagonal matrices

$$
\phi\left(a^{1} e_{1}+\ldots+a^{6} e_{6}\right)=\operatorname{diag}\left(R\left(a^{1} \theta_{1}^{j}+\ldots+a^{6} \theta_{6}^{j}\right)\right)_{j=1, \ldots, 16},
$$

where $R(\theta)$ is the $2 \times 2$ matrix representing rotation by $2 \pi \theta$. We extend to a representation of $\Gamma_{\mathbb{R}}=\Gamma \otimes \mathbb{R} \cong \mathbb{R}^{6}$ by letting $a^{i}$ in (7.4) be real numbers. Define the group $G_{\mathbb{R}}$ as the fiber product of $P$ and $G$ over $S O(6)$ by the maps $\varrho_{P}$ and $j$; it is the pullback of the group extension (7.1) via the map $\varrho_{P}$. There is a commutative diagram

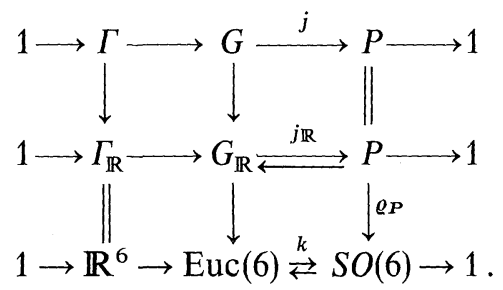

Note that the splitting map in (7.1) pulls back to the splitting $P \rightarrow G_{\mathbb{R}}$. Now each element $\tilde{g} \in G_{\mathbb{R}}$ can be written as $v \cdot g$ for some $v \in \Gamma_{\mathbb{R}}, g \in G$. We define $\phi_{\mathbb{R}}(\tilde{g})$ $=\phi(v) \phi(g)$. [Recall that (7.4) defines $\phi(v)$.] An easy check shows that this definition is consistent. Thus we obtain a representation $\phi_{\mathbb{R}}: G_{\mathbb{R}} \rightarrow S O(32)$. Finally, composing with the splitting map we obtain a representation $\phi_{P}: P \rightarrow S O(32)$ of the finite point group $P$.

Proposition 7.6. For any characteristic class $v$ of $S O(32)$, we have $v(\phi)=j^{*} v\left(\phi_{P}\right)$.

This follows from the commutative diagram (7.5).

Propositions 7.3 and 7.6 combine with Corollary 5.12 to yield

Corollary 7.7. The global anomalies cancel on the orbifold $\mathbb{R}^{10} / G$ if :

$$
\begin{gathered}
w_{2}\left(\varrho_{P}\right)=w_{2}\left(\phi_{P}\right)=0 ; \\
\lambda\left(\varrho_{P}\right)=\lambda\left(\phi_{P}\right) .
\end{gathered}
$$

We remark that this result holds for both abelian and nonabelian point groups. These conditions were also derived in Sect. 6.

Next we derive explicit formulae for the characteristic classes $w_{2}$ and $\lambda$ for representations of cyclic groups. These correspond to the level matching conditions of Sect. 2. For infinite cyclic groups the situation is trivial, as is already implicit in the preceding discussion. We state the result explicitly to emphasize that the level matching conditions for elements of infinite order are vacuous.

Proposition 7.8. Let $\tau: \mathbb{Z} \rightarrow O(d)$ be a representation of an infinite cyclic group. Then $w_{2}(\tau)=\lambda(\tau)=0$

The result is immediate from the fact that $H^{*}(\mathbb{Z})=H^{*}\left(S^{1}\right)$ vanishes for $* \geqq 2$.

The integral cohomology of a finite cyclic group $\mathbb{Z} / n \mathbb{Z}$ is generated by an element $x \in H^{2}(\mathbb{Z} / n \mathbb{Z})$ with $n x=n x^{2}=\ldots=0$. In particular, $H^{4}(\mathbb{Z} / n \mathbb{Z})=\mathbb{Z} / n \mathbb{Z}$ with generator $x^{2}$. Now $H^{2}(\mathbb{Z} / n \mathbb{Z} ; \mathbb{Z} / 2 \mathbb{Z}) \cong \mathbb{Z} / n \mathbb{Z} \otimes \mathbb{Z} / 2 \mathbb{Z}$ with generator $\tilde{x}$, the $\bmod 2$ 
reduction of $x$. This group vanishes for odd $n$ and is cyclic of order 2 for even $n$.

Proposition 7.9. Let $\tau: \mathbb{Z} / n \mathbb{Z} \rightarrow O(d)$ be a representation of $\mathbb{Z} / n \mathbb{Z}$. By conjugation we can assume that $\tau$ maps into the maximal torus of $O(d)$. Let the generator map to an element with rotation angles $2 \pi i r_{j} / n, j=1,2, \ldots,\left[\frac{d}{2}\right]$. Then

$$
w_{2}(\tau)= \begin{cases}\left(\sum_{j} r_{j}\right) \tilde{x}, & \text { for } n \text { even } \\ 0, & \text { for } n \text { odd }\end{cases}
$$

If $w_{2}(\tau)=0$, then $\lambda(\tau)$ is well-defined, and

$$
\lambda(\tau)=\left(\frac{1}{2} * \sum_{j} r_{j}^{2}\right) x^{2}= \begin{cases}\left(\frac{1}{2} \sum_{j} r_{j}^{2}\right) x^{2}, & \text { if } \sum r^{2} \text { is even } \\ \frac{1}{2}\left(\sum_{j} r_{j}^{2}+n\right) x^{2}, & \text { if } \sum r^{2} \text { is odd }\end{cases}
$$

Here $r_{j}$ are determined $\bmod n$. For $n$ odd, multiplication by 2 is invertible, and (7.11) is the formula for the inverse, which we denote $\frac{1}{2} *$. For $n$ even, when $w_{2}(\tau)=0$, then $\sum r_{j}^{2} \equiv \sum r^{j} \equiv 0(\bmod 2)$, and $\frac{1}{2} \sum r_{j}^{2}$ in $\mathbb{Z} / n \mathbb{Z}$ is unambiguously defined.

Proof. Recall that the classifying space for $\mathbb{Z} / n \mathbb{Z}$ is the infinite lens space $L_{n}$. Let $\xi \rightarrow L_{n}$ be the tautological complex line bundle; its total Chern class is $1+x$. The characteristic classes of $\tau$ are the characteristic classes of the bundle $\oplus_{j} \xi^{r_{J}} \rightarrow L_{n}$. By the Whitney sum formula its total Chern class is

$$
\prod_{j}\left(1+r_{j} x\right)=1+\left(\sum_{j} r_{j}\right) x+\left(\sum_{j<k} r_{j} r_{k}\right) x^{2}+\ldots
$$

Since $w_{2}=c_{1}(\bmod 2)$, we obtain (7.10). Also, $p_{1}=c_{1}^{2}-2 c_{2}$, from which

$$
p_{1}(\tau)=\left(\left(\sum r_{j}\right)^{2}-2 \sum_{j<k} r_{j} r_{k}\right) x^{2}=\left(\sum r_{j}^{2}\right) x^{2}
$$

Now we must compute $\lambda(\tau)$. Recall that

$$
2 \lambda(\tau)=p_{1}(\tau)
$$

When $n$ is odd $\lambda(\tau)$ is uniquely determined by (7.12), and is given by (7.11). For $n$ even there are two classes $-\left(\frac{1}{2} \sum r_{j}^{2}\right) x^{2}$ and $\frac{1}{2}\left(\sum r_{j}^{2}+n\right) x^{2}-$ which satisfy (7.12), and we need further argument to eliminate the latter. Now $\tau$ is a complex representation, and since $w_{2}(\tau)=0$ there is a lifted representation $\hat{\tau}$ in the double cover of $U\left(\left[\frac{d}{2}\right]\right)$. However, there are two possible lifts, corresponding to two possible lifts of the generator of $\mathbb{Z} / n \mathbb{Z}$. The double cover of the unitary group has a two dimensional integral characteristic class $\mu$ satisfying $2 \mu=c_{1}$ and $\lambda=2 \mu^{2}-c_{2}$. Now for $n$ even,

$$
\mu(\hat{\tau})=\left(\frac{1}{2} \sum_{j} r_{j}\right) x \quad \text { or } \quad \frac{1}{2}\left(\sum_{j} r_{j}+n\right) x
$$


depending on the lift. But in either case,

$$
2 \mu^{2}(\hat{\tau})=\frac{1}{2}\left(\sum_{j} r_{j}\right)^{2} x^{2}
$$

from which

$$
\lambda(\tau)=2 \mu^{2}(\hat{\tau})-c_{2}(\tau)=\left(\frac{1}{2} \sum_{j} r_{j}^{2}\right) x^{2}
$$

as claimed.

The next proposition shows that for representations of abelian groups these characteristic classes are determined by restricting to finite cyclic subgroups.

Proposition 7.13. Let $\tau: H \rightarrow O(d)$ be a representation of a finitely generated abelian group $H$. Set $v=w_{2}$ or $\lambda$. Suppose that for each finite cyclic subgroup $i: \mathbb{Z} / n \mathbb{Z} \hookrightarrow H$ the characteristic class $i^{*} v(\tau)$ vanishes. Then $v(\tau)=0$.

Proof. Fix an isomorphism $H \cong \mathbb{Z}^{s} \times \mathbb{Z} / n_{1} \mathbb{Z} \times \mathbb{Z} / n_{2} \mathbb{Z} \times \ldots \times \mathbb{Z} / n_{t} \mathbb{Z}$. By Proposition 7.8 the class $v(\tau)$ dies on $\mathbb{Z}^{s}$. Therefore, we restrict our attention to the torsion subgroup of $H$. Then $\tau$ is the realification of a complex representation which decomposes into a sum of one dimensional representations indexed by a $t$-tuple of integers $\left(r^{1}, r^{2}, \ldots, r^{t}\right)$. Denote the generator of $H^{2}\left(\mathbb{Z} / n_{i} \mathbb{Z}\right)$ by $x_{i}$. The total Chern class of $\tau$ is

$$
\prod_{j=1}^{\left[\frac{d}{2}\right]}\left(1+r_{j}^{1} x_{1}+r_{j}^{2} x_{2}+\ldots+r_{j}^{t} x_{t}\right) \text {. }
$$

We see immediately that

$$
w_{2}(\tau)=\left(\sum_{j} r_{j}^{1}\right) \tilde{x}_{1}+\ldots+\left(\sum_{j} r_{j}^{t}\right) \tilde{x}_{t}
$$

The restriction of $w_{2}(\tau)$ to $\mathbb{Z} / n_{i} \mathbb{Z}$ is $\left(\sum_{j} r_{j}^{i}\right) \tilde{x}_{i}$, which vanishes by hypothesis. Therefore, $w_{2}(\tau)=0$. Now from (7.4) we compute

$$
\lambda(\tau)=\sum_{i}\left(\frac{1}{2} \sum_{j}\left(r_{j}^{i}\right)^{2}\right) x_{i}^{2}-\sum_{i_{1}<i_{2}}\left(\sum_{j}^{r_{j}^{i_{1}} r_{j}}\right) x_{i_{1}} x_{i_{2}} .
$$

By restricting to $\mathbb{Z} / n_{i} \mathbb{Z}$ we find that the first term vanishes. To see that the second term is also zero, we restrict to the cyclic subgroup $C$ generated by the product of the generators of $\mathbb{Z} / n_{i_{1}} \mathbb{Z}$ and $\mathbb{Z} / n_{i_{2}} \mathbb{Z}$. Its eigenvalues are $\left\{r_{j}^{i_{1}}+r_{j}^{i_{2}}\right\}_{j}$, so that by (7.11) the restriction of $\lambda(\tau)$ to this subgroup is

$$
\sum_{j}\left(\frac{1}{2}\left(r_{j}^{i_{1}}\right)^{2}+r_{j}^{i_{1}} r_{j}^{i_{2}}+\frac{1}{2}\left(r_{j}^{i_{2}}\right)^{2}\right) y^{2},
$$

where $y$ is the generator in $H^{2}(C)$. The first and last terms vanish, since by hypothesis $\lambda(\tau)$ restricts to zero on $\mathbb{Z} / n_{i_{1}} \mathbb{Z}$ and $\mathbb{Z} / n_{i_{2}} \mathbb{Z}$. Hence the middle term is also zero. It follows that the second term in (7.15) vanishes, completing the proof that $\lambda(\tau)=0$.

The reader may find it instructive to compare this proof with the analogous argument in [1]. There it was seen that the modular invariance of the torus with boundary conditions $(g, h)$ requires level matching not only in the $g$ and $h$ sectors 
but also in the $g h$ sector. The cohomological analogue of this is seen in the above computation.

Recall from Sect. 2 that an element $g \in G$ of finite order $n$ satisfies the level matching conditions if the rotation angles $2 \pi i r_{j} / n, 2 \pi i s_{k} / n$ of $\varrho, \phi$ satisfy

$$
\begin{gathered}
\sum r_{j} \equiv \sum s_{k} \equiv 0(\bmod 2) \quad(\text { for } n \text { even }), \\
\frac{1}{2} * \sum\left(r_{j}\right)^{2} \equiv \frac{1}{2} * \sum\left(s_{k}\right)^{2}(\bmod n) .
\end{gathered}
$$

We can also define level matching conditions for elements of $P$ using the representations $\varrho_{P}$ and $\phi_{P}$. We combine Corollary 7.7 and Propositions 7.9 and 7.13 in

Corollary 7.18. Suppose the point group $P$ is abelian. Then the global anomalies on the orbifold $\mathbb{R}^{10} / G$ cancel if each element of $P$ satisfies the level matching conditions (7.16) and (7.17).

Note that this result is valid whether or not the sequence (7.1) is split, i.e., whether or not the point group sits inside the space group. In case (7.1) is split it suffices to check the level matching conditions for all elements of finite order in the space group $G$. Our topological approach proves (7.18) for Riemann surfaces of arbitrary genus. Corollary 7.18 encompasses the results of Sects. 2 and 3. The necessity of the level matching conditions will be discussed below (cf. [1]).

We turn now to arbitrary nonabelian point groups. For complex representations one can check the vanishing of $w_{2}$ on cyclic subgroups.

Proposition 7.19. Let $\tau$ be a complex representation of a finite group $P .{ }^{21}$ Then $w_{2}(\tau)$ vanishes if the restriction of $\tau$ to each cyclic subgroup has vanishing $w_{2}$. In particular, if $\varrho_{P}$ and $\phi_{P}$ factor through complex representations, then 7.7(i) is satisfied if the level matching condition (7.16) holds for all elements of $P$.

Proof. For complex representations $w_{2}$ has an integral lift $c_{1}$ in second cohomology. Furthermore, $c_{1}(\tau)=c_{1}(\operatorname{det} \tau)$, where $\operatorname{det} \tau$ is the one dimensional representation obtained by taking the determinant. Let $P_{a b}=P /[P, P]$ be the abelianization of $P$. Then since det $\tau$ factors through $P_{a b}$, and every element of $P_{a b}$ lifts to $P$, Proposition 7.13 applies to yield the desired conclusion.

Our next task is to give explicit formulae for the global anomaly in the situations contemplated at the end of Sect. 5. There we first considered a oneparameter family of flat $G$ bundles over a torus. However, in view of Corollary 7.7 it suffices to consider flat $P$ bundles instead. Let $T$ denote the fundamental group of the torus; it is isomorphic to $\mathbb{Z} \times \mathbb{Z}$. A flat $P$ bundle over a torus is determined by a representation $\sigma: T \rightarrow P$. Now suppose that for some $A \in S L(2 ; \mathbb{Z})$ and some $c \in P$ we have $\sigma \circ A=c \sigma c^{-1}$ [cf. (5.13)]. This data determines an extension of $\sigma$ to a representation $\sigma_{K}: K_{A} \rightarrow P$ of the semi-direct product $K_{A}=\mathbb{Z} \ltimes T$, which is a flat $P$ bundle over the classifying space of $K_{A}$. Now since $P$ is a finite group, its rational cohomology vanishes, and so the Bockstein homomorphisms

$$
\beta: H^{i-1}(P ; \mathbb{Q} / \mathbb{Z}) \rightarrow H^{i}(P)
$$

\footnotetext{
${ }^{21}$ More generally, take $\tau$ to be a real representation whose integral third Stiefel-Whitney class $W_{3}(\tau)$ vanishes
} 
are isomorphisms. We denote the unique lift of $v \in H^{i}(P)$ by $v^{\mathbb{Q} / \mathbb{Z}} \in H^{i-1}(P ; \mathbb{Q} / \mathbb{Z})$. Assume that condition (i) in Corollary 7.7 is satisfied. ${ }^{22}$ Then the results of [2] (cf. the discussion following Theorem 5.5) show that the global anomaly around this one-parameter family of tori is given by

$$
\left(\lambda^{\mathbb{Q} / \mathbb{Z}}\left(\varrho_{P} \circ \sigma_{K}\right)-\lambda^{\mathbb{Q} / \mathbb{Z}}\left(\phi_{P} \circ \sigma_{K}\right)\right)\left[K_{A}\right] \in \mathbb{Q} / \mathbb{Z} .
$$

To evaluate (7.21) we give explicit formulae for the first Pontrjagin class of a representation of $K_{A}$. We first treat one dimensional complex representations. Observe that the elements of finite order in $U(1)$ form the group $\mathbb{Q} / \mathbb{Z}$. We write $\bar{u} \in \mathbb{Q} / \mathbb{Z}$ for the corresponding element $e^{2 \pi i u} \in U(1)$, where $u$ is any rational number lifting $\bar{u}$. Let $A^{*}$ denote the transpose of $A$.

Theorem 7.22. Suppose $\tau: T \rightarrow U(1)$ is a representation mapping every element of $T$ to a finite order element in $U(1)$. Assume that $\tau \circ A=\tau$, so that $\tau$ lifts to a representation $\tau_{K}$ of $K_{A}$. Fix an identification $T \cong \mathbb{Z} \times \mathbb{Z}$ and suppose the generators map to $\bar{u}, \bar{v} \in \mathbb{Q} / \mathbb{Z}$. Choose lifts $u, v \in \mathbb{Q}$ and set

$$
\left(\begin{array}{l}
s \\
t
\end{array}\right)=\left(1-A^{*}\right)\left(\begin{array}{l}
u \\
v
\end{array}\right)
$$

Then $\mathrm{s}, \mathrm{t}$ are integers, and

$$
p_{1}^{\mathbb{Q} / \mathbb{Z}}\left(\tau_{K}\right)\left[K_{A}\right]=\overline{s v-t u} \in \mathbb{Q} / \mathbb{Z} .
$$

The following lemmas will aid us in the proof of Theorem 7.22.

Lemma 7.24. Suppose $H$ is a discrete group, and $\tau: H \rightarrow \mathbb{Q} / \mathbb{Z} \subset U(1)$ a one dimensional complex representation sending elements of $H$ into elements of finite order. Then the image of $\tau$ under $\operatorname{Hom}(H, \mathbb{Q} / \mathbb{Z}) \rightarrow H^{1}(H ; \mathbb{Q} / \mathbb{Z})$ maps via the Bockstein $\beta: H^{1}(H ; \mathbb{Q} / \mathbb{Z}) \rightarrow H^{2}(H)$ to the first Chern class $c_{1}(\tau)$.

Proof. The map $\tau$ factors through a homomorphism $\tau: H \rightarrow \mathbb{Z} / n \mathbb{Z}$ for some $n$. Under the Bockstein isomorphism $\beta: H^{1}(\mathbb{Z} / n \mathbb{Z} ; \mathbb{Q} / \mathbb{Z}) \rightarrow H^{2}(\mathbb{Z} / n \mathbb{Z})$ the generator $x \in H^{2}(\mathbb{Z} / n \mathbb{Z})$ corresponds to the inclusion map in $\operatorname{Hom}(\mathbb{Z} / n \mathbb{Z}, \mathbb{Q} / \mathbb{Z})$ $\cong H^{1}(\mathbb{Z} / n \mathbb{Z} ; \mathbb{Q} / \mathbb{Z})$. As $x$ is the first Chern class of the inclusion $\mathbb{Z} / n \mathbb{Z} \hookrightarrow U(1)$, the lemma follows.

Next we relate the cohomology of $K_{A}$ to the cohomology of $T$. In what follows we will use $T, K_{A}$ to denote both the group and the associated classifying space. Recall from Sect. 4 that the classifying space of $T$ is a torus and the classifying space of $K_{A}$ is a torus bundle over the circle. Let the circle be covered by two connected open arcs (overlapping in two disjoint open arcs), and lift to a covering of $K_{A}$. The Mayer-Vietoris sequence (or spectral sequence) for this covering yields the following exact sequences:

$$
\begin{aligned}
& 0 \longrightarrow \mathbb{Z} \stackrel{\delta^{*}}{\longrightarrow} H^{1}\left(K_{A}\right) \stackrel{i_{*}}{\longrightarrow} H^{1}(T) \stackrel{1-A^{*}}{\longrightarrow} H^{1}(T) \stackrel{\delta^{*}}{\longrightarrow} \\
& \stackrel{\delta^{*}}{\longrightarrow} H^{2}\left(K_{A}\right) \stackrel{i^{*}}{\longrightarrow} H^{2}(T) \longrightarrow 0, \\
& 0 \longrightarrow H^{2}(T) \stackrel{\delta^{*}}{\longrightarrow} H^{3}\left(K_{A}\right) \longrightarrow 0 .
\end{aligned}
$$

${ }^{22}$ If not, then we are forced into a more delicate calculation in $K$-theory 
Here $i: T \rightarrow K_{A}$ is the inclusion (of a fiber). These sequences hold for $\mathbb{Z}, \mathbb{Q}$, and $\mathbb{Q} / \mathbb{Z}$ coefficients.

As in the previous lemma we identify $\tau$ in Theorem 7.22 with a cocycle in $C^{1}(T ; \mathbb{Q} / \mathbb{Z})$ and with the associated cohomology class. Choose a rational cochain $\tilde{\tau} \in C^{1}(T ; \mathbb{Q})$ whose reduction $\bmod \mathbb{Z}$ is $\tau$. We claim that $\left(1-A^{*}\right) \tilde{\tau}$ is closed, i.e., $\delta\left(\left(1-A^{*}\right) \tilde{\tau}\right)=0$. To see this view $A$ as a diffeomorphism of the torus; then the induced action on cochains commutes with the differential. Since $\operatorname{det} A=1$, it follows that $1-A^{*}$ is zero on 2-cochains, from which $\delta \circ\left(1-A^{*}\right)$ $=\left(1-\operatorname{det} A^{*}\right) \circ \delta=0$, verifying our claim.

Lemma 7.27. In the situation of Theorem 7.22, we have

$$
\begin{gathered}
i^{*}\left(\tau_{K}\right)=\tau ; \\
\delta^{*}\left(\left(1-A^{*}\right) \tilde{\tau}\right)=c_{1}\left(\tau_{K}\right) .
\end{gathered}
$$

Proof. Equation (7.28) simply asserts that the representation $\tau_{K}$ restricts on $T$ to the representation $\tau$. Now $\left(1-A^{*}\right) \tilde{\tau}$ is an integral cocycle, because $\tau \circ A=\tau$ by hypothesis. However, we use rational coefficients to compute $\delta^{*}\left(\left(1-A^{*}\right) \tilde{\tau}\right)$. Following the definition of the connecting homomorphism, consider the 2-cocycle $\delta \tilde{\tau} \in C^{2}(T ; \mathbb{Q})$. Since $1-A^{*}$ acts trivially on 2 -cochains, this lifts to a 2 -cocycle $c \in C^{2}\left(K_{A} ; \mathbb{Q}\right)$. But $c$ is actually an integral cocycle, because its reduction $\bmod \mathbb{Z}$ is $\delta \tau$, which vanishes. To deduce (7.29) we use the commutative diagram

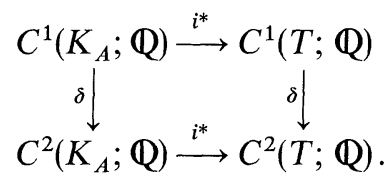

Let $\tilde{\tau}_{K} \in C^{1}\left(K_{A} ; \mathbb{Q}\right)$ be a lift of $\tau_{K}$ mapping to $\tilde{\tau}$ under $i^{*}$. Then (7.30) implies

$$
i^{*}\left(\delta \tilde{\tau}_{K}\right)=\delta \tilde{\tau} .
$$

Furthermore, both $\delta \tilde{\tau}_{K}$ and $\delta \tilde{\tau}$ are integral cocycles, whence (7.31) holds over the integers. Now (7.29) follows from Lemma 7.24 and the definition of the Bockstein homomorphism.

Proof of Theorem 7.22. First, since $\tau_{K}$ is a one dimensional representation, $p_{1}\left(\tau_{K}\right)$ $=c_{1}\left(\tau_{K}\right)^{2}$. Now $\tau_{K}$ factors through a finite cyclic group, so we can factor $\tau_{K}$ through the projection $K_{A} \rightarrow \bar{K}_{A}$ onto $\bar{K}_{A}=\mathbb{Z} / m \mathbb{Z} \ltimes_{A}(\mathbb{Z} / n \mathbb{Z} \ltimes \mathbb{Z} / n \mathbb{Z})$ for some $m, n$. By Lemma 7.24 we have $c_{1}^{\mathbb{Q} / \mathbb{Z}}\left(\tau_{K}\right)=\tau_{K}$ [cf. (7.20)], whence

$$
\beta\left(\tau_{K} \smile c_{1}\left(\tau_{K}\right)\right)=c_{1}\left(\tau_{K}\right)^{2},
$$

since the Bockstein $\beta$ respects cup products. It follows that $p_{1}^{\mathbb{Q} / \mathbb{Z}}\left(\tau_{K}\right)=\tau_{K} \smile c_{1}\left(\tau_{K}\right)$. Let $\partial^{*}: H_{3}\left(K_{A}\right) \rightarrow H_{2}(T)$ be the adjoint of $\delta^{*}$ in (7.26); then $\partial^{*}\left[K_{A}\right]=[T]$. Therefore, by (7.32) and Lemma 7.27,

$$
\begin{aligned}
p_{1}^{\mathbb{Q} / \mathbb{Z}}\left(\tau_{K}\right)\left[K_{A}\right] & =\left(\tau_{K} \smile c_{1}\left(\tau_{K}\right)\right)\left[K_{A}\right]=-\delta^{*}\left(\tau \smile\left(1-A^{*}\right) \tilde{\tau}\right)\left[K_{A}\right] \\
& =-\left(\tau \smile\left(1-A^{*}\right) \tilde{\tau}\right)[T] .
\end{aligned}
$$


Relative to our chosen basis we have $\tau=\left(\begin{array}{l}\bar{u} \\ \bar{v}\end{array}\right)$ and $\left(1-A^{*}\right) \tilde{\tau}=\left(\begin{array}{l}s \\ t\end{array}\right)$, whence

$$
-\left(\tau \smile\left(1-A^{*}\right) \tilde{\tau}\right)[T]=\overline{s v-t u} \in \mathbb{Q} / \mathbb{Z},
$$

as claimed.

The next result extends Theorem 7.22 to higher dimensional representations. Formula (7.36) agrees with (3.4) after squaring the latter.

Theorem 7.34. Let $\tau: T \rightarrow U(\ell)$ be a homomorphism whose image consists of finite order transformations. By conjugation we can ensure that $\tau(T)$ consists of diagonal matrices. Assume there exists a permutation $\pi$ on $\ell$ letters such that $\tau \circ A=\pi \circ \tau \circ \pi^{-1}$, and denote the resulting representation of $K_{A}$ by $\tau_{K}$. Fix an identification $T \cong \mathbb{Z} \times \mathbb{Z}$, and suppose the generators map to $\operatorname{diag}\left(\bar{u}_{j}\right), \operatorname{diag}\left(\bar{v}_{j}\right)$. Choose lifts $u_{j}, v_{j} \in \mathbb{Q}$ and set

$$
\left(\begin{array}{l}
s_{j} \\
t_{j}
\end{array}\right)=\left(\begin{array}{l}
u_{\pi^{-1}(j)} \\
v_{\pi^{-1}(j)}
\end{array}\right)-A^{*}\left(\begin{array}{l}
u_{j} \\
v_{j}
\end{array}\right) \text {. }
$$

Then $s_{j}, t_{j}$ are integers, and

$$
p_{1}^{\mathbb{Q} / \mathbb{Z}}\left(\tau_{K}\right)\left[K_{A}\right]=\sum_{j} \overline{s_{j} v_{j}-t_{j} u_{j}}
$$

Proof. Consider the one dimensional representation $\operatorname{det} \tau_{K}$. The generators map to $\sum \bar{u}_{j}, \sum \bar{v}_{j} \in \mathbb{Q} / \mathbb{Z}$, and Theorem 7.22 implies

$$
\left(c_{1}\left(\tau_{K}\right)^{2}\right)^{\mathbb{Q} / \mathbb{Z}}\left[K_{A}\right]=\left(c_{1}\left(\operatorname{det} \tau_{K}\right)^{2}\right)^{\mathbb{Q} / \mathbb{Z}}\left[K_{A}\right]=\sum_{j, k} \overline{s_{k} v_{j}-t_{k} u_{j}}
$$

To calculate $c_{2}^{\mathbb{Q} / \mathbb{Z}}\left(\tau_{K}\right)$ we use the following device. Let $\Delta: T \rightarrow T^{\ell}$ be the diagonal inclusion, and define $\eta: T^{\ell} \rightarrow U(\ell)$ as the homomorphism carrying the $j^{\text {th }}$ pair of generators into $\operatorname{diag}\left(0, \ldots, \bar{u}_{j}, \ldots, 0\right), \operatorname{diag}\left(0, \ldots, \bar{v}_{j}, \ldots, 0\right)$; then $\tau=\Delta^{*} \eta$. Now the transformation $\hat{A}=A \otimes \pi^{-1}$ on $T^{\ell}=T \otimes \mathbb{Z}^{\ell}$ preserves $\eta$ by hypothesis: $\eta \circ \hat{A}=\eta$. Thus $\eta$ extends to a representation $\eta_{\hat{K}}$ of $\widehat{K_{A}}=\mathbb{Z} \ltimes_{\hat{A}} T^{\ell}$, and $\Delta$ extends to an inclusion $\hat{\Delta}: K_{A} \rightarrow \widehat{K_{A}}$. Furthermore, $\tau_{K}=\widehat{\Delta}^{*} \eta_{\hat{K}}$. Most importantly, $\eta_{\hat{K}}$ splits into a direct sum of one dimensional representations $\eta_{\hat{K}}=\oplus \eta_{\hat{K}}^{(j)}$. The Whitney sum formula and the naturality of the Bockstein imply

$$
c_{2}^{\mathbb{Q} / \mathbb{Z}}\left(\eta_{\hat{K}}\right)=\sum_{j<k} c_{1}^{\mathbb{Q} / \mathbb{Z}}\left(\eta_{\hat{K}}^{(j)}\right) \smile c_{1}\left(\eta_{\hat{K}}^{(k)}\right)=\sum_{j>k} c_{1}^{\mathbb{Q} / \mathbb{Z}}\left(\eta_{\hat{K}}^{(j)}\right) \smile c_{1}\left(\eta_{\hat{K}}^{(k)}\right)
$$

or

$$
2 c_{2}^{\mathbb{Q} / \mathbb{Z}}\left(\eta_{\hat{K}}\right)=\sum_{j \neq k} c_{1}^{\mathbb{Q} / \mathbb{Z}}\left(\eta_{\hat{K}}^{(j)}\right) \smile c_{1}\left(\eta_{\hat{K}}^{(k)}\right) .
$$

The arguments of the previous theorem extend to show

$$
c_{1}^{\mathbb{Q} / \mathbb{Z}}\left(\eta_{\hat{K}}^{(j)}\right) \smile c_{1}\left(\eta_{\hat{K}}^{(k)}\right)\left[K_{A}\right]=\overline{s_{k} v_{j}-t_{k} u_{j}} .
$$

Finally, (7.36) follows easily from (7.37)-(7.39) and $p_{1}=c_{1}^{2}-2 c_{2}$.

Theorem 7.34 is easily modified to accommodate real representations $\tau: T \rightarrow O(d)$. Since $T$ is abelian we may assume that $\tau(T)$ is contained in the maximal torus of $O(d)$. But now $\pi$ is an element of the Weyl group of $O(d)$, which consists of permutations and sign changes. The modification for sign changes comes in (7.35), 
where if a sign change occurs in the $j^{\text {th }}$ diagonal block, we replace $\left(\begin{array}{l}u_{\pi^{-1}(j)} \\ v_{\pi^{-1}(j)}\end{array}\right)$ with $\left(\begin{array}{l}-u_{\pi^{-1}(j)} \\ -v_{\pi^{-1}(j)}\end{array}\right)$. The proof proceeds via complexification.

Next, we calculate the more refined characteristic class $\lambda$. The reader should compare with (3.4), which is the transformation law for Klein forms. The only issue here is the determination of a sign.

Corollary 7.40. If in the situation of Theorem 7.34 we have $w_{2}\left(\tau_{K}\right)=0$, then

$$
\lambda^{\mathbb{Q} / \mathbb{Z}}\left(\tau_{K}\right)\left[K_{A}\right]=\frac{1}{2} \sum \overline{s_{j}+t_{j}+s_{j} t_{j}}+\frac{1}{2} \sum \overline{s_{j} v_{j}-t_{j} u_{j}} .
$$

Proof. For convenience we treat one dimensional representations, and so adopt the notation of Theorem 7.22; the higher dimensional case is similar. Recall from the proof of Proposition 7.9 that since $w_{2}\left(\tau_{K}\right)=0, \tau_{K}$ lifts to a representation $\hat{\tau}_{K}$ in the double cover of the unitary group. It has a two dimensional integral characteristic class $\mu$ with $2 \mu=c_{1}$ and $\lambda=\left(\mu \smile c_{1}\right)-c_{2}$. Now $\hat{\tau}_{K}$ restricts to a lift $\hat{\tau}$ of $\tau$. Since $c_{1}^{\mathbb{Q} / \mathbb{Z}}(\tau)=\left(\begin{array}{c}\bar{u} \\ \bar{v}\end{array}\right)$, we must have $\mu^{\mathbb{Q} / \mathbb{Z}}(\hat{\tau})=\frac{1}{2}\left(\begin{array}{c}\bar{u}+\delta \\ \bar{v}+\varepsilon\end{array}\right)$, where $\delta, \varepsilon=0$ or 1 . Furthermore, since $\hat{\tau}$ extends to a representation of $K_{A}$, the vector $\frac{1}{2}\left(1-A^{*}\right)\left(\begin{array}{c}\bar{u}+\delta \\ \bar{v}+\varepsilon\end{array}\right)$ consists of integer entries. This implies

$$
\left(1-A^{*}\right)\left(\begin{array}{l}
\delta \\
\varepsilon
\end{array}\right) \equiv\left(\begin{array}{l}
s \\
t
\end{array}\right)(\bmod 2) \text {. }
$$

That such a choice of $\delta, \varepsilon$ is possible follows from (7.25) and (7.29), using the fact that $c_{1}\left(\tau_{K}\right) \equiv 0(\bmod 2)$. Now by $(7.33)$ we have $($ in $\mathbb{Q} / \mathbb{Z})$

$$
\begin{aligned}
\lambda^{\mathbb{Q} / \mathbb{Z}}\left(\tau_{K}\right)\left[K_{A}\right] & =\left(\mu^{\mathbb{Q} / \mathbb{Z}}\left(\hat{\tau}_{K}\right) \smile c_{1}\left(\tau_{K}\right)\right)\left[K_{A}\right]=-\left(\mu^{\mathbb{Q} / \mathbb{Z}}(\hat{\tau}) \smile\left(1-A^{*}\right) \hat{\tau}\right)[T] \\
& =\frac{1}{2}(\overline{s \varepsilon+t \delta})+\frac{1}{2}(\overline{s v-t u}) .
\end{aligned}
$$

It remains to show

$$
s \varepsilon+t \delta \equiv s+t+s t(\bmod 2)
$$

Consider the $\mathbb{Z} / 2 \mathbb{Z}$ quadratic form $q\left(\left(\begin{array}{l}s \\ t\end{array}\right)\right)=s+t+s t$ on the two dimensional $\mathbb{Z} / 2 \mathbb{Z}$-vector space $H^{1}(T ; \mathbb{Z} / 2 \mathbb{Z})$. It is the unique quadratic refinement of the intersection form with Arf invariant 1. Furthermore, it is preserved by all $A \in S L(2 ; \mathbb{Z})$. [This is equivalent to the statement that the distinguished spin structure on the torus is preserved by all $A \in S L(2 ; \mathbb{Z})$.] Hence for any $v \in H^{1}(T ; \mathbb{Z} / 2 \mathbb{Z}$ ), we obtain (in $\mathbb{Z} / 2 \mathbb{Z}$ )

$$
q\left(\left(1+A^{*}\right) v\right)=q(v)+q\left(A^{*} v\right)+v \cdot A^{*} v=2 q(v)+v \cdot A^{*} v=v \cdot A^{*} v
$$

Setting $v=\left(\begin{array}{l}\delta \\ \varepsilon\end{array}\right)$ we obtain the desired equality (7.43).

Following these ideas we have the following computation for higher genus Riemann surfaces [cf., the discussion surrounding (5.15)]. 
Theorem 7.44. Let $\Sigma$ be a Riemann surface of genus $g$, and suppose $\tau: \pi_{1} \Sigma \rightarrow U(1)$ is a representation whose image lies in $\mathbb{Q} / \mathbb{Z}$. Assume that $\tau \circ \varphi=\tau$ for some $\varphi \in$ Diff $\Sigma$. Let $\varphi_{\#}$ denote the induced action on the fundamental group. Then $\tau$ lifts to a representation $\tau_{K}$ of $K=\mathbb{Z} \ltimes_{\varphi_{*}} \pi_{1} \Sigma$. Now since $U(1)$ is abelian, $\tau$ factors through a representation $\bar{\tau}: H_{1} \Sigma \rightarrow U(1)$, and $\bar{\tau}$ is preserved by $A \in \operatorname{Sp}\left(H_{1} \Sigma\right)$, where $A$ is the transformation on homology induced by $\varphi$. Fix a canonical basis for $H_{1} \Sigma$, and suppose the generators map to $\bar{u}_{j}, \bar{v}_{j} \in \mathbb{Q} / \mathbb{Z}$. Fix lifts $u_{j}, v_{j} \in \mathbb{Q}$ and set

$$
\left(\begin{array}{l}
s_{j} \\
t_{j}
\end{array}\right)=\left(\begin{array}{l}
u_{\pi^{-1}(j)} \\
v_{\pi^{-1}(j)}
\end{array}\right)-A^{*}\left(\begin{array}{l}
u_{j} \\
v_{j}
\end{array}\right) \text {. }
$$

Then

$$
p_{1}^{\mathbb{Q} / \mathbb{Z}}\left(\tau_{K}\right)[K]=\sum \overline{s_{j} v_{j}-t_{j} u_{j}} .
$$

Note that this expression agrees with (2.3), which was calculated from the transformation law for the theta function.

To express the formula for $\lambda^{\mathbb{Q} / \mathbb{Z}}\left(\tau_{K}\right)[K]$ we need to find a quadratic refinement $q$ of the intersection pairing on $\Sigma$ which is fixed by $\varphi$. The existence of such a $q$ is guaranteed by [21, Lemma 5.1]. (The relationship between spin structures and quadratic forms is reviewed in [37].)

Corollary 7.45. In the situation of Theorem 7.44 let $q$ be a quadratic refinement of the intersection pairing on $H_{1} \Sigma$ which is preserved by $\varphi$. Then if $w_{2}\left(\tau_{K}\right)=0$, we have

$$
\lambda^{\mathbb{Q} / \mathbb{Z}}\left(\tau_{K}\right)[K]=\frac{1}{2} q\left(\left(\begin{array}{l}
\bar{s} \\
\bar{t}
\end{array}\right)\right)+\frac{1}{2} \sum \overline{s_{j} v_{j}-t_{j} u_{j}} .
$$

We remark that in the setup of Sect. 5 the diffeomorphism $\varphi$ actually preserves a spin structure, hence a quadratic form. It would be interesting to exhibit explicitly the equality of this formula to (2.2), which is the transformation law for the theta function.

The necessity of the level matching conditions for the absence of global anomalies in Corollary 7.18 is a consequence of these formulae. For simplicity we assume that $w_{2}\left(\varrho_{P}\right)=w_{2}\left(\phi_{P}\right)=0$, i.e., that $(7.16)$ holds.

Corollary 7.46. For any $G$ the level matching conditions (7.17) are necessary for the cancellation of global anomalies on $\mathbb{R}^{10} / G$.

Proof. Let $g \in P$ be an arbitrary element, say of order $n$. Then in (5.14) we choose $h=1$ and $\left(\begin{array}{ll}a & c \\ b & d\end{array}\right)=\left(\begin{array}{ll}1 & n \\ 0 & 1\end{array}\right)$. Let $2 \pi i r_{j} / n, 2 \pi i s_{k} / n$ be the eigenvalues of $\varrho_{P}, \phi_{P}$. Then

$$
\begin{aligned}
& \left(1-A^{*}\right)\left(\begin{array}{c}
r_{j} / n \\
0
\end{array}\right)=\left(\begin{array}{c}
0 \\
-r_{j}
\end{array}\right), \\
& \left(1-A^{*}\right)\left(\begin{array}{c}
s_{k} / n \\
0
\end{array}\right)=\left(\begin{array}{c}
0 \\
-s_{k}
\end{array}\right),
\end{aligned}
$$

so that by Corollary 7.40 the global anomaly (7.21) reduces to

$$
\frac{1}{2} \sum\left(r_{j}+s_{j}\right)+\frac{1}{2} \sum\left(r_{j}^{2}-s_{k}^{2}\right) / n(\bmod 1) .
$$

But (7.47) vanishes if and only if the level matching condition (7.17) hold for $g$.

This is exactly the proof which appears in [1]. 


\section{Nonabelian Examples}

In this section we describe some examples. In the previous sections we have already discussed the case where the point group is abelian. Here we consider non-abelian point groups. We show an example of a non-abelian point group with representations satisfying level matching conditions, but which nevertheless suffer from global anomalies. Also, we give some non-abelian examples where level matching conditions are sufficient to guarantee modular invariance.

1. Extra-Special p-Group. Consider a group $G$ generated by three elements $g, h, c$ defined by the following relations

$$
g^{p}=h^{p}=c^{p}=1, \quad g h=h g, \quad c g c^{-1}=g, \quad c h c^{-1}=g h .
$$

We will consider the case of $p=5$ to be specific (but what we shall say applies do any odd $p \geqq 5$ ). Let us consider a five dimensional representation of $G$ described by

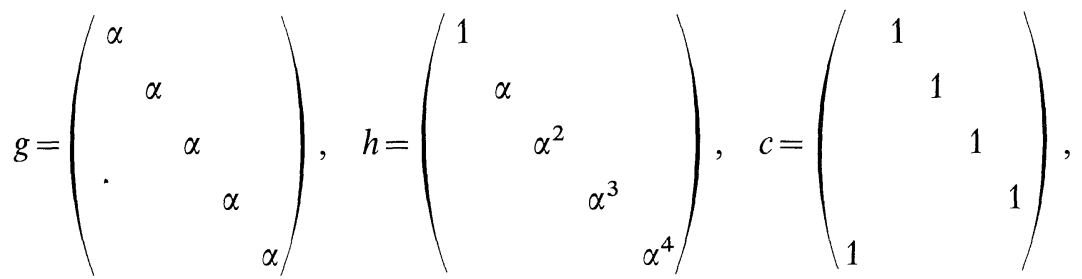

where $\alpha=e^{2 \pi i / 5}$. Let us take this to act only on the gauge fermions. It is easy to see that level matching conditions are satisfied for all elements in the group $\left(\sum r^{2}=0 \bmod 5\right)$. If we consider the torus with twisted boundary conditions given by $(h, g)$, by the arguments in previous sections it should give the same path integral result as $\left(c h c^{-1}, c g c^{-1}\right)=(g h, h)$. This means that under modular transformation $\tau \rightarrow \tau+1$ which is represented by the $S L(2, Z)$ matrix

$$
\left(\begin{array}{ll}
1 & 0 \\
1 & 1
\end{array}\right),
$$

the path integral result for $(h, g)$ should not change. Using the formula given in Sect. 3, we see that the phase for this transformation is given by $(-1) e^{i \pi / 5}$ and therefore the theory is inconsistent due to global anomalies (if one is bothered by the existence of the zero modes of the determinant, we can always consider antiperiodic boundary conditions in the $\tau$ direction).

The cohomology of $G$ is computed in [38]. The result for the four dimensional cohomology is

$$
H^{4}(G) \cong \mathbb{Z} / p \mathbb{Z} \oplus \mathbb{Z} / p \mathbb{Z} \oplus \mathbb{Z} / p \mathbb{Z} \oplus \mathbb{Z} / p \mathbb{Z}
$$

The generators are easily described. First, there is a surjection $G \rightarrow \mathbb{Z} / p \mathbb{Z} \times \mathbb{Z} / p \mathbb{Z}$ obtained by sending $g$ to 1 . Now $H^{4}(\mathbb{Z} / p \mathbb{Z} \times \mathbb{Z} / p \mathbb{Z}) \cong(\mathbb{Z} / p \mathbb{Z})^{3}$ maps injectively to $H^{4}(G)$, which accounts for three of the generators. Notice that these generators are detected on cyclic subgroups (generated by $h, c$, and $h c$ ). Since an appropriate $K_{A}$ maps the fundamental class onto the generator of the third homology of a cyclic 
group [cf., (7.46)], these three generators are realized by some $K_{A}$. We claim that the remaining generator is realized by the $K_{A}$ described in the previous paragraph. For the calculation there shows that the fundamental class of $K_{A}$ maps nontrivially to $H_{3}(G)$, to an element of order $p$. But the first Pontrjagin class of the representation (8.2) vanishes when restricted to the cyclic group generated by $h, c$, or $h c$. So $K_{A}$ represents the remaining generator of $H_{3}(G)$, and all of $H^{4}(G)$ is detected completely by the groups $K_{A}$.

2. Dihedral Group of Order 8. Now we will consider an example which has already come up in physics, and that is the non-supersymmetric $E_{8}$ theory [39]. In the fermionic formulation, this corresponds to modding out the gauge degrees of freedom by a dihedral group of order 8 , denoted by $D_{4}$, which is the symmetries of the square (see, for example, [32]). We will show that in this case the level matching conditions are sufficient to guarantee modular invariance. The representation of this group which comes up in physics, is sixteen copies of the standard two dimensional real representation of $D_{4}$ acting on the square which is the subgroup of $S O(32)$. In fact, the minimum number of representations necessary for levelmatching is 8 , so let us consider that case. The group $D_{4}$ is generated by two elements $r, \theta$, with $r^{2}=\theta^{4}=1$, and $r \theta r^{-1}=\theta^{-1}$. Consider the commuting pair $\left(r, \theta^{\dot{2}}\right)$. We can conjugate these with the element $\theta$, and we get

$$
\theta\left(r, \theta^{2}\right) \theta^{-1}=\left(r \theta^{2}, \theta^{2}\right),
$$

so there is a potential for anomaly under $\tau \rightarrow \tau+1$, but this time it is easy to check that there is no phase picked up. So eight copies of the representation passes the consistency check.

From the cohomology point of view the arguments of the previous paragraph assert that under the inclusion of the union of cyclic subgroups into $D_{4}$, the induced map on $\mathrm{H}^{4}$ is injective. In other words, all of $\mathrm{H}^{4}\left(D_{4}\right)$ is detected on cyclic subgroups. This is easily seen from [40, p. 192].

3. Quaternion Group of Order 8. A more instructive example is the quaternion group $Q=\{ \pm 1, \pm i, \pm j, \pm k\}$. For any representation of $Q$ the only thing to check at one loop beyond level matching is that, for example, $(i,-i)$ gives the same contribution as $(-i, i)$ (by conjugation by $j$ ). But that is manifest because the two boundary conditions can be obtained from the $S L(2 ; \mathbb{Z})$ matrix which is minus the identity. That does not act on the modulus of the torus, and so there is no problem with modular invariance at one loop. It is not difficult to see that all the potential problems with anomalies at one loop are of this type, and so we cannot get any further condition apart from level matching.

Now it is well-known that $H^{4}(Q) \cong \mathbb{Z} / 8 \mathbb{Z}[41$, p. 254]. $Q$ contains cyclic subgroups of order 4 , and any inclusion $\mathbb{Z} / 4 \mathbb{Z} \hookrightarrow \mathbb{Z} / 8 \mathbb{Z}$ induces an injection on $H_{3}$. So twice a generator of $H_{3}(Q)$ is represented by some $K_{A}$. On the other hand, the generator is not so represented (Proposition 8.5 below). Hence the nontrivial element of order 2 in $H^{4}(G)$ cannot be detected by any $K_{A}$.

Let $\tau$ denote the nontrivial 2 dimensional complex representation of $Q$ into $S U(2)$. (The $i, j, k$ map to Pauli matrices.) Now $c_{1}(\tau)=0$ since $\operatorname{det} \tau$ is the trivial representation. 
Proposition 8.3. $\lambda(\tau)=-c_{2}(\tau)$ has order 8 in $H^{4}(Q) \cong \mathbb{Z} / 8 \mathbb{Z}$.

Proof. Set $A=\left(\begin{array}{rr}0 & -1 \\ 1 & 2\end{array}\right)$ and define a map $e: K_{A} \rightarrow Q$ which takes $f_{1} \mapsto j, f_{2} \mapsto j^{-1}$, $f \mapsto i$. Here $f_{1}, f_{2}$ are the generators of $\mathbb{Z} \times \mathbb{Z}$ and $f$ generates $\mathbb{Z}$ in $K_{A}=\mathbb{Z} \ltimes_{A}(\mathbb{Z} \times \mathbb{Z})$. In the notation of Theorem 7.34 we can take $u_{1}=1 / 4$, $v_{1}=-1 / 4, u_{2}=-1 / 4, v_{2}=1 / 4$, and $\pi$ is the transposition $1 \leftrightarrow 2$. Hence $s_{1}=0$, $t_{1}=1, s_{2}=0, t_{2}=-1$. So from Corollary 7.40 we compute $\left\langle\lambda^{\mathbb{Q} / \mathbb{Z}}(\tau), e_{*}\left[K_{A}\right]\right\rangle$ $=-1 / 4$. By the arguments in the preceding paragraph $e_{*}\left[K_{A}\right]$ is divisible by 2 in $H_{3}(Q)$. It follows that $\lambda(\tau)$ has order 8 .

Of course, this means that $p_{1}(\tau)=-2 c_{2}(\tau)$ has order exactly 4 .

Consider the representation $4 \tau$. By Proposition 8.3 we have $\lambda(4 \tau)=4 \lambda(\tau)$ is the nontrivial element of order 2 in $H^{4}(Q)$. Since the image of $\left[K_{A}\right]$ under any homomorphism $K_{A} \rightarrow Q$ is divisible by 2 , this image pairs trivially with $\lambda(4 \tau)$. Hence $4 \tau$ is an example of a representation with $\lambda \neq 0$ yet not detectable by a torus bundle over a circle. (The representation $2 \tau$ provides a similar example for $p_{1}$.) Therefore, the characteristic class $\lambda$ (or $p_{1}$ ) is not always detectable by some $K_{A}$. Hence we have shown

Proposition 8.4. There exists a representation of a finite group for which the characteristic class $\lambda$ is nonzero, yet evaluates to zero on the image of any $K_{A}$.

We would like to know whether (in this example) $\lambda$ is detected by a Riemann surface bundle over the circle, but we could not determine it. Our best result in that direction is ${ }^{23}$

Proposition 8.5. Let $\pi$ denote the fundamental group of a Riemann surface $\Sigma$ of genus $g$, and $\varphi$ an element of the mapping class group. Then $\varphi$ acts as an automorphism of $\pi$, and we form the semidirect product $L_{\varphi}=\mathbb{Z} \ltimes_{\varphi} \pi$. Its classifying space is a 3-manifold whose fundamental class we denote $\left[L_{\varphi}\right]$. Suppose e $: L_{\varphi} \rightarrow Q$ is a homomorphism and e maps $\pi$ into some $\mathbb{Z} / 4 \mathbb{Z} \subset Q$. Then $4 e_{*}\left[L_{\varphi}\right]=0$ in $H_{3}(Q)$.

Note that the hypothesis on $e$ is always satisfied if $\Sigma$ is a torus.

Proof. Write $L_{\varphi}$ as the split extension

$$
1 \rightarrow \pi \rightarrow L_{\varphi} \leftrightarrows \mathbb{Z} \rightarrow 1
$$

and $Q$ as the extension

$$
1 \rightarrow \mathbb{Z} / 4 \mathbb{Z} \rightarrow Q \rightarrow \mathbb{Z} / 2 \mathbb{Z} \rightarrow 1 .
$$

The map $e: L_{\varphi} \rightarrow Q$ induces $f: \mathbb{Z} \rightarrow \mathbb{Z} / 2 \mathbb{Z}$, via the splitting in (8.6), and our hypotheses imply that $e$ is a map of group extensions. (We can assume that $f$ is nontrivial; otherwise, e maps $L_{\varphi}$ into $\mathbb{Z} / 4 \mathbb{Z}$ and the proposition follows immediately.) The argument now proceeds by comparing the homology spectral sequences of (8.6) and (8.7).

Because $B \mathbb{Z}=S^{1}$ and $B \pi=\Sigma$ are manifolds of dimensions 1 and 2, respectively, the spectral sequence $E_{p q}^{2}=H_{p}\left(\mathbb{Z}, H_{q}(\pi)\right) \Rightarrow E_{p q}^{\infty}=\operatorname{Gr} H_{p+q}\left(L_{\varphi}\right)$ is concentrated in

${ }^{23}$ This argument was worked out jointly with John Morgan 
the rectangle $0 \leqq p \leqq 1,0 \leqq q \leqq 2$. Hence $H_{3}\left(L_{\varphi}\right)=E_{12}^{\infty}=E_{12}^{2}=\mathbb{Z}$. Note that the filtration on $H_{3}\left(L_{\varphi}\right)$ induced by the spectral sequence is

$$
\begin{aligned}
& F_{0} \subset F_{1}=H_{3}\left(L_{\varphi}\right) \\
& \|\quad\| \\
& 0 \quad \mathbb{Z} .
\end{aligned}
$$

The spectral sequence $E_{p q}^{\prime 2}=H_{p}\left(\mathbb{Z} / 2 \mathbb{Z}, H_{q}(\mathbb{Z} / 4 \mathbb{Z})\right) \Rightarrow E_{p q}^{\prime \infty}=\mathrm{Gr} H_{p+q}(Q)$ is more complicated. The action of $\mathbb{Z} / 2 \mathbb{Z}$ on $\mathbb{Z} / 4 \mathbb{Z}$ is nontrivial, and the invariants in low dimensional homology are $H_{1}(\mathbb{Z} / 4 \mathbb{Z})^{\mathbb{Z} / 2 \mathbb{Z}}=\mathbb{Z} / 2 \mathbb{Z}, H_{3}(\mathbb{Z} / 4 \mathbb{Z})^{\mathbb{Z} / 2 \mathbb{Z}}=\mathbb{Z} / 4 \mathbb{Z}$. Hence the $E^{\prime 2}$ term starts off

\begin{tabular}{l|cccc}
3 & $\mathbb{Z} / 4 \mathbb{Z}$ & & & \\
2 & 0 & 0 & & \\
1 & $\mathbb{Z} / 2 \mathbb{Z}$ & $\mathbb{Z} / 2 \mathbb{Z}$ & $\mathbb{Z} / 2 \mathbb{Z}$ & \\
0 & $\mathbb{Z}$ & $\mathbb{Z} / 2 \mathbb{Z}$ & 0 & $\mathbb{Z} / 2 \mathbb{Z}$ \\
\hline & 0 & 1 & 2 & 3
\end{tabular}

Since $H_{3}(Q)=\mathbb{Z} / 8 \mathbb{Z}$ we see that $d^{\prime 2}: E_{30}^{\prime 2} \rightarrow E_{12}^{\prime 2}$ is an isomorphism. Hence $E_{03}^{\prime \infty}=\mathbb{Z} / 4 \mathbb{Z}, E_{12}^{\prime \infty}=0, E_{21}^{\prime \infty}=\mathbb{Z} / 2 \mathbb{Z}, E_{30}^{\prime \infty}=0$. The induced filtration on $H_{3}(Q)$ is then

$$
\begin{array}{ccccccc}
F_{0}^{\prime} & \subset & F_{1}^{\prime} & \subset & F_{2}^{\prime} & \subset & F_{3}^{\prime}=H_{3}(Q) \\
\| & & \| & & \| & \| \\
\mathbb{Z} / 4 \mathbb{Z} & \mathbb{Z} / 4 \mathbb{Z} & & \mathbb{Z} / 8 \mathbb{Z} & \mathbb{Z} / 8 \mathbb{Z} .
\end{array}
$$

The argument is now clear. The map $e_{*}: H_{3}\left(L_{\varphi}\right) \rightarrow H_{3}(Q)$ preserves the filtrations. Then from (8.8) and (8.9) it follows that the image of $e_{*}$ lies in $\mathbb{Z} / 4 \mathbb{Z} \subset H_{3}(Q)$, and so consists of elements of order at most 4 .

Unfortunately, we were unable to determine whether the generator of $H_{3}(Q)$ is represented by a surface bundle (of genus $>1$ ) over a circle.

Motivated by the constraints in constructing the Hilbert space (Sect. 3) and the examples above, we formulate the following question: can all global anomalies be detected at 1-loop? In other words, if for a given finite group $P$ and representation $\tau$ [with $w_{2}(\tau)=0$ ] we can find a map $K=\mathbb{Z} \ltimes_{\varphi} \pi \rightarrow P$ (where $\pi$ is the fundamental group of a Riemann surface and $\varphi$ an outer automorphism) for which $\lambda(\tau)[K] \neq 0$, then can we also find a map $K_{A}=\mathbb{Z} \ltimes_{A}(\mathbb{Z} \times \mathbb{Z}) \rightarrow P$ for which $\lambda(\tau)\left[K_{A}\right] \neq 0$ ? Here $B K$ is a Riemann surface bundle over a circle and $B K_{A}$ is a torus bundle over a circle.

In this paper we have derived sufficient conditions for the absence of global anomalies. We can ask whether these cohomology constraints are also necessary for the absence of global anomalies. We strongly suspect that the answer is no. In fact, the example of the quaternion group discussed above most likely provides a definitive answer. However, we can ask whether other physical principles (beyond anomalies) necessitate these cohomology conditions. We understand that work of Killingback potentially answers this question in the affirmative. The new physical ingredient is factorization of the amplitudes. Factorization entails understanding how the determinant bundle behaves at the boundary of moduli space. 


\section{References}

1. Vafa, C.: Modular invariance and discrete torsion on orbifolds. Nucl. Phys. B 273, 592 (1986)

2. Freed, D.S.: Determinants, torsion, and strings. Commun. Math. Phys. 107, 483-513 (1986)

3. Gross, D.J., Harvey, J.A., Martinec, E., Rohm, R.: Heterotic string theory (I). The free heterotic string. Nucl. Phys. B 256, 253 (1985)

4. Dixon, L., Harvey, J., Vafa, C., Witten, E.: Strings on orbifolds (II). Nucl. Phys. B 274, 285 (1986)

5. Narain, K.S., Sarmadi, M.H., Vafa, C.: Asymmetric orbifolds. Harvard University preprint, HUTP-86/A089

6. Kawai, H., Lewellen, D.C., Tye, S.-H.H.: Phys. Rev. Lett. 57, 1832 (1986); Cornell preprint CLNS 86/751

7. Meuller, M., Witten, E.: Princeton preprint (1986)

8. Antoniadis, I., Bachas, C., Kounnas, C.: To appear

9. Witten, E.: Global anomalies in string theory. In: Anomalies, geometry, and topology. White, A. (ed.). World Scientific 1985, pp. 61-99

10. Wen, X.-G., Witten, E.: Electric and magnetic charges in superstring models. Princeton preprint

11. Witten, E.: Global gravitational anomalies. Commun. Math. Phys. 100, 197-229 (1985)

12. Witten, E.: Topological tools in ten dimensional physics. Lectures at the Workshop on Unified String Theories, Santa Barbara

13. Atiyah, M.F.: The logarithm of the Dedekind $\eta$-function. Preprint

14. Kubert, D.S., Lang, S.: Modular units. Berlin, Heidelberg, New York: Springer 1981

15. Freed, D.S.: On determinant line bundles. In: Mathematical aspects of string theory. Yau, S.T. (ed.). New York: World Scientific (to appear)

16. Frenkel, I., Lepowsky, J., Meurmen, A.: In: Proceedings of a conference on vertex operators in mathematics and physics. Lepowsky, J. (ed.). Berlin, Heidelberg, New York: Springer 1984

17. Landweber, P.S., Ravenel, D., Stong, R.: Periodic cohomology theories defined by elliptic curves. Preprint

18. Witten, E.: Elliptic genera and quantum field theory. Preprint

19. Alvarez-Gaumé, L., Witten, E.: Gravitational anomalies. Nucl. Phys. B 234, 269 (1983)

20. Lang, S.: Elliptic functions. Berlin, Heidelberg, New York: Springer 1973

21. Atiyah, M.F.: Riemann surfaces and spin structures. Ann. Scient. Éc. Norm. Sup. 4e série, t. 4, fasc. 1, 47-62 (1971)

22. Alvarez-Gaumé, L., Moore, G., Vafa, C.: Theta functions, modular invariance, and strings. Commun. Math. Phys. 106, 1 (1986)

23. Mumford, D.: Tata lectures on theta, Vols. I and II. Boston: Birkhäuser 1983

24. Igusa, J.: Theta functions. Berlin, Heidelberg, New York: Springer 1972

25. Bott, R.: Lectures on Morse theory, old and new. Bull. AMS 7, 331-358 (1982)

26. Atiyah, M.F., Bott, R.: The moment map and equivariant cohomology. Topology 23, 1-28 (1984)

27. Atiyah, M.F., Singer, I.M.: Dirac operators coupled to vector potentials. Proc. Nat. Acad. Sci. 81, 259 (1984)

28. Quillen, D.: Determinants of Cauchy-Riemann operators over a Riemann surface. Funk. Anal. iprilozen 19, 3 (1985)

29. Bismut, J.M., Freed, D.S.: The analysis of elliptic families: Metrics and connections on determinant bundles. Commun. Math. Phys. 106, 159-176 (1986)

30. Bismut, J.M., Freed, D.S.: The analysis of elliptic families: Dirac operators, eta invariants, and the holonomy theorem of Witten. Commun. Math. Phys. 107, 103-160 (1986)

31. Narain, K.S., Sarmadi, M.H., Witten, E.: Nucl. Phys. B 279, 369 (1987)

32. Ginsparg, P., Vafa, C.: Toroidal compactification of non-supersymmetric heterotic strings. Harvard University preprint HUTP-86/A064 
33. Atiyah, M.F., Patodi, V.K., Singer, I.M.: Spectral asymmetry and Riemannian geometry. I. Math. Proc. Cambridge Philos. Soc. 77, 43-69 (1975); II, Math. Proc. Cambridge Philos. Soc. 78, 405-432 (1975); III, Math. Proc. Cambridge Philos. Soc. 79, 71-99 (1976)

34. Alvarez-Gaumé, L., Ginsparg, P.: The structure of gauge and gravitational anomalies. Ann. Phys. 161, 423 (1985)

35. Elitzur, S., Nair, V.: Non-perturbative anomalies in higher dimensions. Nucl. Phys. B 243, 205 (1984)

36. Pontrjagin, L.: In: Topological groups. London, New York, Paris: Gordon and Breach 1966, Chap. 1

37. Lee, R., Miller, E.Y., Weintraub, S.H.: Spin structures: their relationship to quadratic pairings, Arf invariants, Rohlin invariants, and the holonomy of determinant line bundles, preprint

38. Lewis, G.: The integral cohomology rings of groups of order $p^{3}$. Trans. AMS 132, 501-529 (1968)

39. Dixon, L., Harvey, J.: String theories in ten dimensions without spacetime supersymmetry. Nucl. Phys. B 274, 93 (1986)

40. Evens, L.: On the Chern classes of representations of finite groups. Trans. AMS 108, 180-193 (1965)

41. Cartan, H., Eilenberg, S.: Homological algebra. Princeton: Princeton University Press 1956

Communicated by L. Alvarez-Gaumé

Received January 28, 1987 
